\title{
UNC-31 (CAPS) Is Required for Dense-Core Vesicle But Not Synaptic Vesicle Exocytosis in Caenorhabditis elegans
}

\author{
Sean Speese, ${ }^{1 \star}$ Matt Petrie, ${ }^{2 \star}$ Kim Schuske, ${ }^{1}$ Michael Ailion, ${ }^{1}$ Kyoungsook Ann, ${ }^{2}$ Kouichi Iwasaki, ${ }^{3}$ Erik M. Jorgensen, ${ }^{1}$ \\ and Thomas F. J. Martin ${ }^{2}$

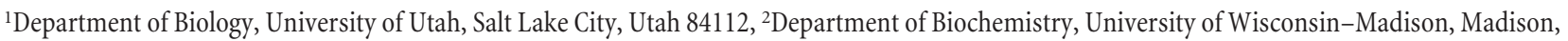 \\ Wisconsin 53706, and ${ }^{3}$ Department of Molecular Pharmacology and Biological Chemistry, Northwestern University Medical School, Chicago, Illinois 60611
}

Previous studies indicated that CAPS (calcium-dependent activator protein for secretion) functions as an essential component for the $\mathrm{Ca}^{2+}$-dependent exocytosis of dense-core vesicles in neuroendocrine cells. However, recent mouse knock-out studies suggested an alternative role in the vesicular uptake or storage of catecholamines. To genetically assess the functional role of CAPS, we characterized the sole Caenorhabditis elegans CAPS ortholog UNC-31 (uncoordinated family member) and determined its role in dense-core vesiclemediated peptide secretion and in synaptic vesicle recycling. Novel assays for dense-core vesicle exocytosis were developed by expressing a prepro-atrial natriuretic factor-green fluorescent protein fusion protein in C. elegans. unc-31 mutants exhibited reduced peptide release in vivo and lacked evoked peptide release in cultured neurons. In contrast, cultured neurons from unc-31 mutants exhibited normal stimulated synaptic vesicle recycling measured by FM4-64 [N-(3-triethylammoniumpropyl)-4-(6-(4-diethylamino)phenyl) hexatrienyl)pyridinium dibromide] dye uptake. Conversely, UNC-13, which exhibits sequence homology to CAPS/UNC-31, was found to be essential for synaptic vesicle but not dense-core vesicle exocytosis. These findings indicate that CAPS/UNC-31 function is not restricted to catecholaminergic vesicles but is generally required for and specific to dense-core vesicle exocytosis. Our results suggest that CAPS/ UNC-31 and UNC-13 serve parallel and dedicated roles in dense-core vesicle and synaptic vesicle exocytosis, respectively, in the $C$. elegans nervous system.

Key words: CAPS; UNC-13; dense-core vesicle; exocytosis; synaptic vesicle; $C$. elegans

\section{Introduction}

Neurotransmitters are secreted from neurons by two types of vesicles. Small clear synaptic vesicles contain classical transmitters such as glutamate, GABA, and acetylcholine that activate postsynaptic ionotropic receptors and mediate fast synaptic transmission. Dense-core vesicles contain neuropeptides and monoamines that bind and activate G-protein-coupled receptors and modulate presynaptic or postsynaptic function. There are many similarities between synaptic vesicle and dense-core vesicle exocytosis. Both classes of vesicles require $\mathrm{Ca}^{2+}$ to trigger exocytosis, and several proteins [e.g., SNAREs (soluble $N$ ethylmaleimide-sensitive factor attachment protein receptor), Munc-18, and synaptotagmin] are common required constitu-

Received Nov. 20, 2006; revised April 25, 2007; accepted April 28, 2007.

This work was supported by grants from the McKnight Foundation, United States Department of Agriculture Cooperative State Research, Education, and Extension Service Project WIS04664, and National Institutes of Health (NIH) Grants DK40428 (T.F.J.M.) and MH60997 (E.M.J.). E.M.J. is an investigator of the Howard Hughes Medical Institute. We thank Jamie White for aid in worm tracking and Steve Fields for providing advice and guidance on the FM4-64 dye uptake studies in cultured neurons. We thank Ken Miller for sharing data regarding the molecular nature of unc-31(e928) before publication. We thank Patrick Hullet for sequencing of unc-31 CDNAs. We additionally thank Mark Alkema for use of laboratory space and reagents and for critical feedback on this manuscript. We acknowledge T. E. Johnson, M. Chalfie, H. R. Horvitz, J. Hodgkin, M. Nonet, and the Caenorhabditis Genetics Center for providing strains.

*S.S. and M.P. contributed equally to this work.

Correspondence should be addressed to Thomas F. J. Martin, Department of Biochemistry, University of Wisconsin-Madison, Madison, WI 53706. E-mail: tfmartin@wisc.edu.

DOI:10.1523/JNEUROSCI.1466-07.2007

Copyright $\odot 2007$ Society for Neuroscience $\quad$ 0270-6474/07/276150-13\$15.00/0 ents for the fusion of either vesicle type (Rettig and Neher, 2002; Jahn et al., 2003; Sorensen, 2004). Despite similarities in the fusion machinery for both vesicle types, there are differences in the kinetics of exocytosis and in the physiological regulation of release (Rettig and Neher, 2002; Martin, 2003). These differences suggest that there are proteins and mechanisms that are distinct for synaptic vesicle and dense-core vesicle exocytosis.

CAPS1 (calcium-dependent activator protein for secretion) was isolated from brain cytosol as a factor that restored $\mathrm{Ca}^{2+}$ triggered dense-core vesicle exocytosis in permeable neuroendocrine cells (Martin and Walent, 1989; Walent et al., 1992; Ann et al., 1997; Grishanin et al., 2004). CAPS1 is present on dense-core but not synaptic vesicles in brain homogenates (Berwin et al., 1998), and synaptosome studies revealed a requirement for CAPS1 in $\mathrm{Ca}^{2+}$-triggered catecholamine secretion but not $\mathrm{Ca}^{2+}$ dependent glutamate release (Tandon et al., 1998). On this basis, it was suggested that CAPS1 functions specifically in dense-core but not synaptic vesicle exocytosis, but conclusive genetic tests of this proposal remain to be conducted.

A study of a $C A P S 1^{-/-} / C A P S 2^{+/+}$mouse concluded that CAPS1 is instead essential for the uptake or storage of catecholamines in dense-core vesicles (Speidel et al., 2005). However, a role for CAPS1 in vesicle exocytosis could not be directly assessed because of redundancy with CAPS2. In contrast, a study of a CAPS $1^{+/+} / \mathrm{CAPS}^{-/-}$mouse found strong deficits in densecore vesicle-mediated neurotrophin secretion in the cerebellum 
A

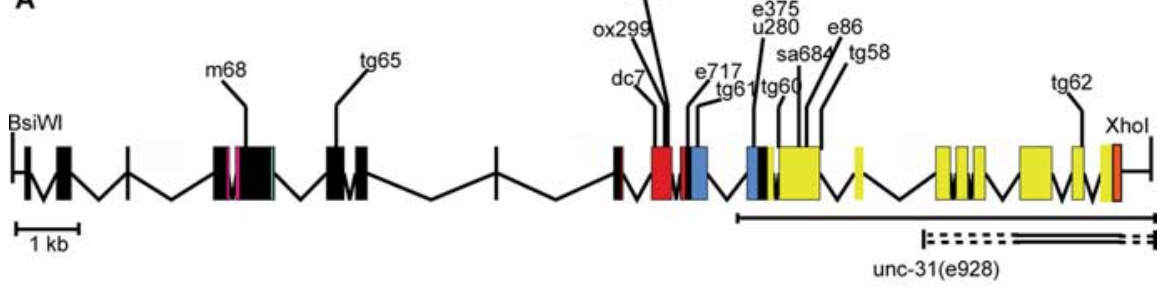

B

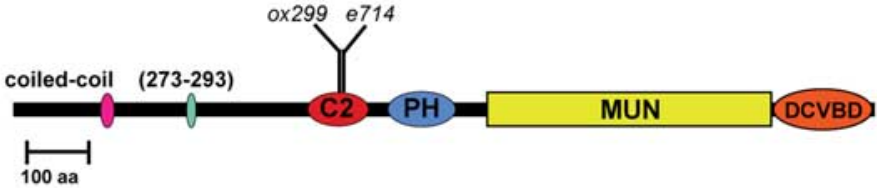

C

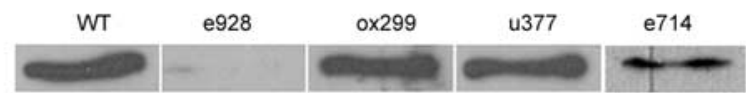

D C2 domain

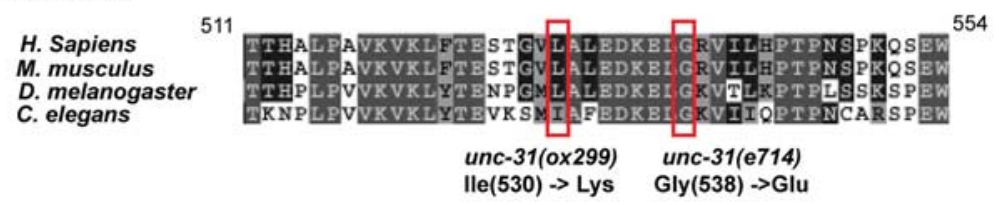

E GFP fusion constructs

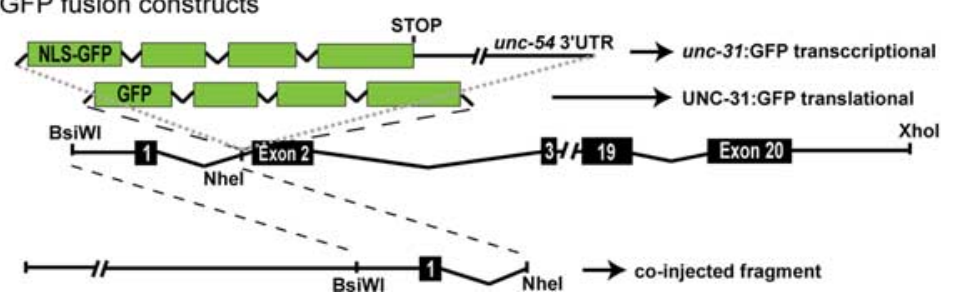

Figure 1. Genomic structure, mutant allele characterization, and protein architecture for UNC-31. A, Schematic representation of the UNC-31 genomic structure. For details for unc-31 mutant alleles, see Table 1. All alleles are nonsense or splice site mutations except $0 \times 299$, e714, and e928. 0x299 corresponds to the missense mutation 1530K and e 714 corresponds to the missense mutation G538E. e928 is a deletion indicated by solid black line; the double bar underneath represents a transposition of a fragment of the unc-31 locus detected in the e 928 allele. $B$, UNC-31 protein architecture. Identified domains in the protein depicted are as follows: coiled-coil (residues 133-159); 20 aa domain defined by alleles u377 and sa534 (273-293) (Table 1); (2 domain (460 -558); and PH domain (582-689C). The coiled-coil, C2, and PH domain were defined by Simple Modular Architecture Research Tool. The MUN domain (730-1180) (Basu et al., 2005) was defined by multiple alignment programs. The dense-core vesicle binding domain (DCVBD) (1181 end) was defined based on alignment and Grishanin et al. (2002). C, Western blotting for UNC-31. Homogenates (100 $\mu \mathrm{g}$ of protein) from wild-type (WT) and mutant strains were analyzed by Western blotting with an affinity-purified UNC-31 antibody. Results shown are representative of at least two independent experiments. unc-37(e714) exhibited decreased UNC-31 levels ( $22 \pm 13 \%$ of wild type, mean $\pm \mathrm{SE} ; n=3$ ). $\boldsymbol{D}$, Alignment of protein sequences for a portion of the $\mathrm{C} 2$ domain of $C$. elegans UNC-31 with those from human, mouse, and Drosophila orthologs. Highlighted in the red box are the residues mutated in unc-31(0x299) and unc-31(e714). E, Schematic representation of unc-31::GFP transcriptional and translational constructs and an overlapping fragment that was coinjected. Coinjection was done to provide additional promoter elements that were necessary for full rescue (S. Speese, unpublished observations). UTR, Untranslated region; NLS, nuclear localization sequence.

in which CAPS2 predominates (Sadakata et al., 2007a). In Drosophila melanogaster in which a single gene encodes $d C A P S$, a null mutant was found to exhibit mild reductions in synaptic transmission (Renden et al., 2001). These were thought to be secondary to more primary defects in the secretion of modulatory transmitters, but a direct assay for dense-core vesicle exocytosis was not available. Thus, uncertainties about the precise role of CAPS in vesicle secretory pathways motivate additional genetic studies.

Because the unc-31 (uncoordinated family member) gene in Caenorhabditis elegans encodes the single CAPS ortholog in the worm (Livingstone, 1991; Ann et al., 1997; Charlie et al., 2006), we analyzed CAPS/ UNC-31 function in C. elegans. We developed a novel in situ assay for peptide secretion and novel cultured neuron assays for peptide secretion and synaptic vesicle recycling. With these, we directly assessed whether CAPS is required for regulated peptide secretion and whether CAPS functions selectively for dense-core vesicle exocytosis or also for synaptic vesicle exocytosis. We found that CAPS is expressed throughout the nervous system, that unc-31 mutants developed normally, and that CAPS/UNC-31 was required for the regulated release of dense-core vesicle peptide contents but not for evoked synaptic vesicle recycling. Conversely, UNC13, which exhibits sequence homology to CAPS, was required for synaptic vesicle cycling but not for dense-core vesicle exocytosis.

\section{Materials and Methods}

Strains. The following mutant strains were used: EG3404 unc-31(e928), EG3405 unc31(u280), and BC168 unc-13(s69). The green fluorescent protein (GFP)-expressing strains used in this study were as follows: EG3344 oxIs180[Paex-3:ANF::GFP], EG3346 unc-31(e928) oxIs207[Paex-3:ANF::GFP], EG3680 oxIs206[Paex3:ANF::GFP], EG3681 unc-31(e928) oxIs206[Paex3:ANF::GFP], EG3682 unc-31(u280) oxIs206[Paex3:ANF:: GFP], EG3345 unc-13(s69) oxIs206[Paex3:ANF:: GFP], EG3683 unc-13(e51) oxIs206[Paex3:ANF:: GFP], EG3410 oxEx608 [Punc-31:GFP], EG3407 unc-31(e928) oxEx605[Punc-31: UNC-31::GFP], EG1285 oxIs12[Punc-47:GFP], EG1846 unc-31(u280); oxIs12[Punc-47:GFP], MT8247 lin-15(n765) nIs52[Punc-25:SNB-1:: GFP; lin-15(+)], EG1845 unc-31(u280) nIs52[Punc-25:SNB-1::GFP; lin-15(+)], and EG2623 unc-31(e928); lin-15(n765ts) oxEx93 [Pacr-5::SNB::GFP; lin-15(+)]. Additional strains used include the following: EG2844 unc31(e928) oxIs79[hsp:UNC-31].

Molecular biology. The Punc-31 GFP transcriptional and UNC-31::GFP translational constructs (Fig. 1E) were generated from a common vector called pSS1.5. To construct pSS1.5, a $15.5 \mathrm{~kb}$ AleI-XhoI fragment containing the entire $u n c-31$ intron/exon structure and $\sim 2 \mathrm{~kb}$ of upstream sequence was cut from cosmid ZK897 and ligated into the EcoRV-XhoI sites of pLitmus28 (New England Biolabs, Beverly, MA). The rescuing Punc-31::UNC-31:: GFP translational construct (pSS1.6) was generated by cutting an $\mathrm{XbaI}$ fragment containing the open reading frame for GFP without a stop codon, and flanked by splice acceptor and donor sequences, from the vector pPD114.24 (provided by A. Fire, Stanford University, Palo Alto, $\mathrm{CA})$. The $\mathrm{Xba \textrm {I }}$ fragment was ligated into a unique NheI site in pSS1.5 which lies in the first intron of unc-31. The Punc-31::GFP transcriptional construct (pSS1.7) was constructed by PCR amplification of the GFP open reading frame preceded by a nuclear localization sequence and ending with a stop codon, followed by the unc-54 $3^{\prime}$ untranslated region from the vector pPD95-67. Oligonucleotides for the PCR added a 3' 
Table 1. unc-31 alleles

\begin{tabular}{|c|c|c|}
\hline Allele & Molecular nature & Protein \\
\hline unc-31(u280) & $Q(680) \rightarrow$ STOP & No \\
\hline unc-31(e928) & GACAAAAA. . . . . TGAATGTT (see legend) & No \\
\hline unc-31(ox299) & $\mathrm{I}(530) \rightarrow \mathrm{K}$ & Yes \\
\hline unc-31(tg58) & Disrupts 5' splice donor of intron 13 & ND \\
\hline unc-31(tg60) & Disrupts 3' splice acceptor of intron 12 & No \\
\hline unc-31(tg61) & $Q(611) \rightarrow$ STOP & No \\
\hline unc-31(tg62) & $\mathrm{R}(1,260) \rightarrow$ STOP & No \\
\hline unc-31(tg64) & Unknown breakpoints & ND \\
\hline unc-31(tg65) & $Q(330) \rightarrow$ STOP & No \\
\hline unc-31(m68) & $R(193) \rightarrow$ STOP & No \\
\hline unc-31(n577) & Disrupts 5' splice donor of intron 5 & No \\
\hline & Disrupts $5^{\prime}$ splice donor of intron 5 and & \\
\hline unc-31(u375) & deletion of exon 14 & No \\
\hline unc-31(z1) & Disrupts 5 ' splice donor of intron 5 & Yes \\
\hline unc-31(u377) & Disrupts $5^{\prime}$ splice donor of intron 5 & Yes \\
\hline unc-31(sa534) & Disrupts $5^{\prime}$ splice donor of intron 5 & Yes \\
\hline unc-31(e169) & Disrupts 5 ' splice donor of intron 9 & No \\
\hline unc-31(sa349) & Disrupts 5 ' splice donor of intron 9 & No \\
\hline unc-31(dc7) & $W(503) \rightarrow$ STOP & No \\
\hline unc-31(e714) & $\mathrm{G}(538) \rightarrow \mathrm{E}$ & Yes \\
\hline unc-31(n1304) & Disrupts 3' splice acceptor of intron 10 & No \\
\hline unc-31(n422) & Disrupts 3 ' splice acceptor of intron 10 & No \\
\hline unc-31(e717) & $\mathrm{R}(550) \rightarrow$ STOP & ND \\
\hline unc-31(e69) & $Q(680) \rightarrow$ STOP & ND \\
\hline unc-31(e375) & $Q(680) \rightarrow S T O P$ & ND \\
\hline unc-31(sa684) & W (754) $\rightarrow$ STOP & No \\
\hline unc-31(e86) & Q (821) $\rightarrow$ STOP & No \\
\hline
\end{tabular}

ND, Not determined.

splice acceptor at the $5^{\prime}$ end of the product and $X b a \mathrm{I}$ cleavage sites at both the $5^{\prime}$ and $3^{\prime}$ ends. The PCR product was digested with XbaI and ligated into the unique NheI site of pSS1.5. Primers used in the PCR were as follows: forward, GCTCTAGATTTTCAGATGACTGCTCCAAAGAAGAAGCG; and reverse, GCTCTAGAAACTTACAAACAGTTATGTTTGGTATATTGGGAATGTATTC. The Paex-3:ANF::GFP-4 plasmid was generated by subcloning an atrial natriuretic factor (ANF)enhanced GFP construct (generously provided by E. Levitan, University of Pittsburgh, Pittsburgh, PA) into a pBSII SK + plasmid containing an aex-3 (aBoc, expulsion defective) promoter.

Characterization of unc- 31 alleles. unc-31 alleles were characterized by either sequencing PCR amplification products from genomic DNA or reverse transcription (RT)-PCR from mRNA. For RT-PCR, poly $\left(\mathrm{A}^{+}\right)$ RNA was prepared, and four amplicons spanning the $4106 \mathrm{bp}$ coding sequence of UNC-31 mRNA were generated for sequencing. The $e 928$ allele was demonstrated to be a $5.2 \mathrm{~kb}$ deletion (Livingstone, 1991). PCR analysis demonstrated that the locus deletes regions encoding the C-terminal half of the protein and that part of the deletion, including the last three exons, are inserted at an unknown location in the genome. As summarized in Figure $1 A$ and Table 1, we molecularly characterized 26 alleles of $u n c-31$. Isolation of additional alleles was performed by a noncomplementation screen with unc-31(e928). Briefly, N-ethyl-Nnitrosourea-mutagenized males were crossed with L4 $d p y$-20(e2017) unc-31(e928) hermaphrodites, and F1 progeny were screened for a nonDpy (Dumpy), sluggish Unc phenotype. Unc F1 progeny were allowed to self-propagate, and Unc non-Dpy F2 progeny were picked. F3 progeny were screened using the unc-31 pumping constitutive phenotype by loading nonstarved worms with a 1:20 v/v suspension of Fluoresbrite microspheres (Polysciences, Warrington, PA) for 6 min (Avery et al., 1993).

UNC-31 antibody and Western blotting. DNA corresponding to amino acids 42-304 of UNC-31 coding sequence was amplified and cloned into a pQE30 (Invitrogen, Carlsbad, CA) vector for expression in Escherichia coli strain JM109. Protein was purified on a Ni-NTA column (Invitrogen) and used to immunize rabbits. Immune sera were purified by adsorption to and elution from polyvinylidene difluoride blots containing the fusion protein and further adsorbed on acetone powders prepared from unc-31(e928) animals. Homogenates for Western blotting were prepared by resuspending well washed worms in nematode solubilization buffer ( $0.3 \%$ ethanolamine, 2 mM EDTA, and 5 mM DTT), followed by microwaving on high for $80 \mathrm{~s}$. Homogenates were mixed with $2 \times$ SDS-PAGE sample buffer [with Roche Diagnostics (Mannheim, Germany) protease inhibitor cocktail], boiled, and centrifuged at $16,000 \times g$, and supernatants were analyzed by SDS-PAGE, followed by transfer to nitrocellulose membranes for sequential incubation with UNC-31 antibody and protein A/HRP for ECL (Pierce, Rockford, IL) detection.

Strain construction. Transgenic strains were generated using standard microinjection techniques (Mello et al., 1991). Punc-31::GFP transcriptional and UNC-31::GFP translational strains were generated by coinjection with an overlapping PCR product that included $6.8 \mathrm{~kb}$ additional promoter regions of $u n c$ - 31 (Fig. $1 E$ ) found to be necessary for full rescue. The PCR product encompassed -6798 to +405 bp relative to the unc-31 ATG (called unc-31 promoter PCR fragment). This fragment was digested with NheI and coinjected with the BsiWI digestions of pSS1.6 and pSS1.7 to generate the Punc-31:UNC-31::GFP and Punc-31::GFP arrays, respectively. During array formation, homologous recombination can occur (Mello et al., 1991) between the linear pSS1.7 and pSS1.6 fragments and the $u n c-31$ promoter PCR fragment, which share a $476 \mathrm{bp}$ homology overlap. This recombination event will generate an array with $\sim 6800$ bp of sequence upstream of the ATG of $u n c-31$ with GFP fused in-frame between the first and second exons.

The Punc-31::GFP transcriptional strains were generated by coinjecting pSS1.7 digested with BsiWI (10 ng/ $\mu \mathrm{l})$, the unc-31 promoter PCR fragment (5 ng/ $\mu \mathrm{l})$, Pmyo-2:HIS::GFP (1 ng/ $\mu \mathrm{l} \mathrm{pVil}$; from Susan Mango, University of Utah, Salt Lake City, UT), lin-15(+) (20 ng/ $\mu \mathrm{l})$, and $1 \mathrm{~kb}$ DNA ladder $(64 \mathrm{ng} / \mu \mathrm{l})$ into the wild-type N2 strain. Pmyo-2:HIS:GFP is expressed in the pharynx and is used as a coinjection marker. Three independent stable extrachromosomal lines were recovered: oxEx607, oxEx608, and oxEx609. Note that GFP is inserted in-frame after exon 1, but that the construct contains the entire intron/exon structure of $u n c-31$ downstream of the GFP stop codon.

The following DNA fragments were coinjected into unc-31(e928) animals to generate the UNC-31::GFP translational line: Pmyo-2:HISTONE::GFP (pVi1) $(1 \mathrm{ng} / \mu \mathrm{l}), \operatorname{lin}-15(+)(20 \mathrm{ng} / \mu \mathrm{l}), 1 \mathrm{~kb}$ DNA ladder $(64 \mathrm{ng} / \mu \mathrm{l})$, pSS1.6 (digested with BsiWI and gel purified) $(10 \mathrm{ng} / \mu \mathrm{l})$, and the $u n c-31$ promoter PCR fragment $(5 \mathrm{ng} / \mu \mathrm{l})$. This recombination event will generate a $20 \mathrm{~kb}$ fragment of the full-length $u n c-31$ gene with GFP inserted after amino acid 20. Three independent stable extrachromosomal lines were recovered: oxEx604, oxEx605, and oxEx606.

ANF::GFP lines were generated by injecting lin-15(+) (pbLH98) and Paex-3:ANF::GFP-4 into lin-15(n765) worms. The extrachromosomal array containing Paex-3:ANF::GFP-4 in strain KY5212 was integrated into a chromosome by gamma irradiation and outcrossed four times to generate the allele tgIs5 [Paex-3:ANF::GFP].

unc-31 rescue. Rescue experiments were performed using animals carrying an extrachromosomal array coding for UNC-31::GFP [unc31(e928); oxEx604]. Note that the rescue experiment is not a single gene rescue: there is a U5-10 snRNA in intron 11 and U5-11 snRNA in intron 18 of the unc-31 locus, which are included in the rescue construct. However, it is not likely that these genes are disrupted in the e928 allele because the deletion is $3^{\prime}$ to the U5-10 snRNA and the U5-11 snRNA locus is duplicated and still present in the genome according to our PCR analysis of the $e 928$ allele. To ensure that we are rescuing defects in $u n c-31$ in the unc-31 (e928) allele, we were able to show that unc-31(u280), which does not disrupt either snRNA, has similar phenotypes. In particular, the defecation defects were quantitatively similar (Fig. 2A,B). Additionally, the movement defects of the two alleles were also similar.

Acute expression of $u n c-31$ from a heat shock promoter is able to rescue the anterior body muscle contraction (aBoc) defect in unc31 (e928). Animals were heat shocked at $33^{\circ} \mathrm{C}$ for $1 \mathrm{~h}$ and allowed to recover at room temperature for $6 \mathrm{~h}$. Six cycles were observed for each animal scored to determine the percentage of cycles that had an aBoc event. Heat shock treatment partially rescued the uncoordinated defect of unc-31(e928) animals in the absence of oxIs79, making it difficult to assay locomotion rescue by the transgene.

Movement and defecation cycle analysis. Video tracking was used to 
A

A Percent aBoc per cycle

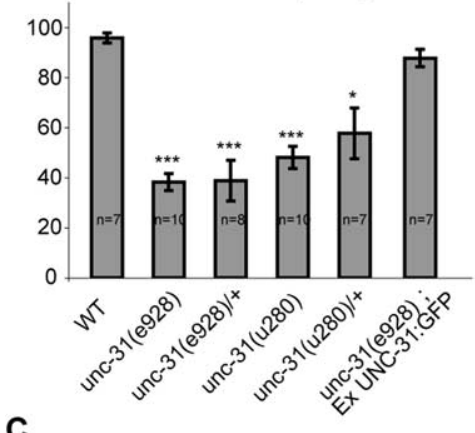

C

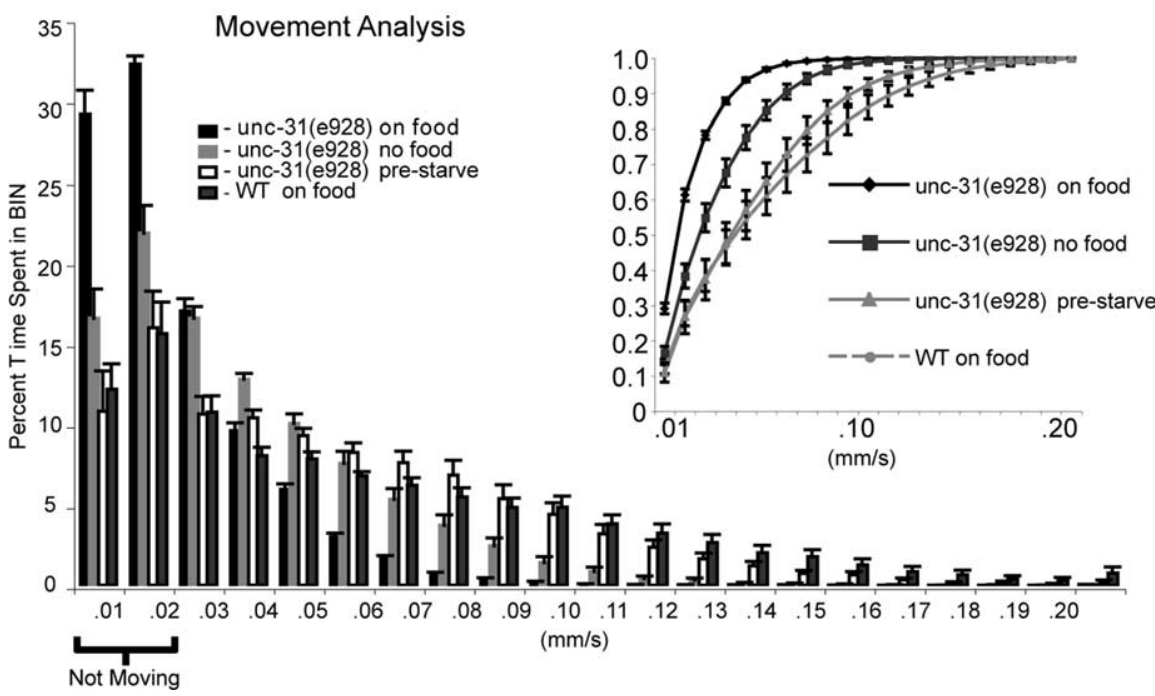

Figure 2. Defecation and movement in unc-31. A, Graph showing the percentage of cycles in which an aBoc was observed in a particular genotype. The number of aBoc events was divided by the total number of cycles scored to generate the percentages. $\boldsymbol{B}$, The number of cycles with an aBoc before an Emc was divided by the number of cycles that contained both an aBoc and an Emc. All worms were scored blind for 10 cycles each. C, Histogram showing the analysis of unc-31(e928) movement on and off food. Velocity (millimeters per second) of the worms was measured for 45 min using a video tracking system and computer analysis. Speeds between 0 and $0.02 \mathrm{~mm} / \mathrm{s}$ were equivalent to the worm not moving. Inset is the same data graphed as a cumulative plot. All animals in these quantitative phenotypic studies were assayed as young adults. In the movement assays, prestarved unc31 (e928) animals were picked as L4s and transferred to a plate without a bacterial lawn for $5 \mathrm{~h}$. Immediately after the starvation period, they were tracked on an assay plate without food. WT, Wild type.

quantify the movement of unc-31 animals under varying food conditions. Plates containing agar were dyed to enhance contrast by top coating with $450 \mu \mathrm{l}$ of $1 \%$ bromophenol blue in S-basal. At 30 min before an assay, plates were spread with a thin layer of HB101 and allowed to dry with the lid partially off. Individual worms were washed in S-basal to clean off residual food. The animals were then picked into $5 \mu$ l drops of S-basal on the plate, and, once dried, video tracking was started and recorded for $45 \mathrm{~min}$. Analysis of velocity was conducted using NIH ImageJ with additional plug-ins written by J. White (University of Utah, Salt Lake City, UT). Captured movies were opened as stacks of eight-bit images and processed to generate a binary image with black worms on a white background. The image stack was used to plot the $x-y$ coordinate of the centroid of every worm every frame ( $1 \mathrm{~s}$ intervals). The $x-y$ coordinates were used to calculate the velocity of individual worms between every frame. All animals assayed were young adult hermaphrodites. Defecation cycles were analyzed by picking L 4 hermaphrodites that were scored blind $12 \mathrm{~h}$ later as young adults. At least 10 cycles were scored for each individual animal. Scoring was recorded using Etho (J. Thomas, University of Washington, Seattle, WA; http://depts.washington.edu/ $\mathrm{jtlab} / \mathrm{software} / \mathrm{softwareIndex.html).} \mathrm{Cycles} \mathrm{were} \mathrm{scored} \mathrm{with} \mathrm{the} \mathrm{lid} \mathrm{of}$ the plate off.

Developmental analysis. All developmental imaging was performed on young adult hermaphrodites. For GABA synapse density analysis, images of the dorsal nerve cord (DNC) were captured at both the anterior and posterior reflex of the gonad for EG1845 unc31(u280); nIs52[Punc-25:SNB-GFP] and MT8247 lin-15(n765ts); nIs52[Punc-25:SNBGFP]. The number of puncta was determined by two methods. In the first, puncta were counted manually from the image. In the second, a line profile was generated along the cord, and peaks were counted on the graph generated by the line profile in NIH ImageJ. The final puncta density for a single animal was calculated by averaging the numbers from both methods of counting for both the images of the anterior and posterior DNC and then taking the average of these two numbers. The number of axons crossing the midline was determined by manually counting the number of axons crossing to and making contact with the DNC in confocal $Z$-series images obtained for strains EG1285 lin-15(n765ts); oxIs12 and EG1846 unc-31(u280); oxIs12. Cell body counts of GABA motor neurons were obtained by manually counting under wide-field fluorescence.

ANF-GFP endocytosis into coelomocytes. Confocal settings used for image capture were held constant from animal to animal and across genotypes. To control for changes in laser power, we monitored the intensity of InSpeck calibration microspheres (Invitrogen) before and after each imaging session. In addition, animals with different genotypes were interlaced within each imaging session. To maintain complete objectivity in sampling of coelomocytes, the cells were identified, and the $Z$-series was established while imaging the cells using bright-field illumination and differential interference contrast (DIC) optics. To control for light scattering issues that could affect the GFP signal collected, only posterior coelomocytes that were not behind other tissues (gut and gonads) and were easily identifiable via DIC were imaged. Collected images were analyzed using the three-dimensional quantitation software Volocity (Improvision, Lexington, MA). Briefly, the total sum of pixel intensities arising from ANF-GFP signal in each coelomocyte was quantitated in three dimensions. This number more closely represents the total amount of ANF-GFP in a particular coelomocyte than the average pixel intensity from a two-dimensional maximum pixel intensity projection. Averages of the total sum of pixel intensities per coelomocyte were used as a basis of comparing wild-type, unc-31, and unc-13 mutants.

Preparation of C. elegans dense-core vesicles and synaptic vesicles. Fluorescent dense-core vesicles and synaptic vesicles were isolated from homogenates of the tgIs5[Paex-3:ANF::GFP] or jsIs219[synaptogyrin::GFP] strains, respectively. Homogenates were prepared from worms on 20 10 -cm nematode growth medium (NGM) plates seeded with E. coli strain NA22 (CGC) and grown to slight starvation. Sucrose-washed worms were resuspended in either cold $0.02 \mathrm{M}$ HEPES, $0.25 \mathrm{M}$ sucrose, $0.002 \mathrm{M}$ EGTA, and protease cocktail (for dense-core vesicles) or a similar buffer with $0.05 \mathrm{M}$ sucrose (for synaptic vesicles) and passed $(\sim 100 \times)$ through a ball homogenizer with a $12 \mu \mathrm{m}$ clearance until cuticles were no longer visible. All subsequent steps were conducted at $4^{\circ} \mathrm{C}$. For dense-core vesicle preparation, the homogenate was clarified by centrifugation at $5000 \times g$, and the supernatant was centrifuged at $100,000 \times g$ for $20 \mathrm{~min}$ in a Ti80 rotor. For synaptic vesicle preparation, the homogenate was centrifuged at 25,000 $\times g$ for $20 \mathrm{~min}$ in a JA20 rotor, and the supernatant was centrifuged at $165,000 \times g$ in a Ti80 rotor for $2 \mathrm{~h}$. Dense-core vesicle and synaptic vesicle pellets were resuspended in corresponding homogenization buffers and fractionated on $0.3-1.5 \mathrm{~m}$ sucrose gradients in a 
SW41 rotor at 28,000 rpm for $90 \mathrm{~min}$. The gradient was collected from the bottom into 16 fractions and subjected to Western blot using a monoclonal anti-GFP antibody (BD Biosciences, San Jose, CA).

Assays for ANF-GFP secretion and FM4-64 dye uptake in cultured neurons. For assays of dense-core vesicle exocytosis in cultured neurons, the tgIs5[Paex-3:ANF::GFP] strain was crossed with unc-31(e928) or unc13(s69) strains to generate cells of the desired genotypes. Embryonic cell cultures were prepared as described previously (Christensen et al., 2002) in coverslip dishes (MatTek, Ashland, MA) and grown in L-15-10 medium for $3-4 \mathrm{~d}$ at $20^{\circ} \mathrm{C}$. Studies were conducted on the stage of a Zeiss (Oberkochen, Germany) Axiovert 100TV inverted microscope fitted with a confocal head (MRC-600; Bio-Rad, Hercules, CA) in a room held at $19^{\circ} \mathrm{C}$. Medium was removed and replaced with either control (in mM: 50 HEPES, $140 \mathrm{NaCl}, 6 \mathrm{KCl}, 10$ glucose, $1 \mathrm{MgCl}_{2}$, and $5 \mathrm{EGTA}, \mathrm{pH} 7.4$ ) or stimulation (in mM: $50 \mathrm{HEPES}, 60 \mathrm{NaCl}, 86 \mathrm{KCl}, 10$ glucose, $1 \mathrm{MgCl}_{2}, 2$ $\mathrm{CaCl}_{2}$, and $5 \mathrm{BaCl}_{2}, \mathrm{pH} 7.4$ ) buffer. Images were recorded using a $63 \times$, 1.4 numerical aperture objective immediately after buffer exchange and at subsequent 30 and $60 \mathrm{~min}$ intervals. Fluorescence intensity in neuronal cell bodies was quantified using MetaMorph software (Universal Imaging, Downingtown, PA).

For assays of synaptic vesicle recycling, the jsIs219[synaptogyrin::GFP] strain was crossed with $u n c-31(e 928)$ or $u n c-13(s 69)$ strains to generate cells of the desired genotypes. Cultured embryonic cells were prepared (Christensen et al., 2002) and grown for $5 \mathrm{~d}$ in L-15-10 medium at $20^{\circ} \mathrm{C}$ in coverslip dishes. Medium was removed, and cells were incubated in depolarization buffer (in mм: $25 \mathrm{HEPES}, 48 \mathrm{KCl}, 2 \mathrm{CaCl}_{2}$, and $2 \mathrm{MgCl}_{2}$, $\mathrm{pH}$ 7.4) containing $5 \mu \mathrm{M}$ FM4-64 [ $N$-(3-triethylammoniumpropyl)-4(6-(4-diethylamino)phenyl)hexatrienyl)pyridinium dibromide] (Invitrogen) for $5 \mathrm{~min}$. Cells were subsequently washed in depolarization buffer without $\mathrm{CaCl}_{2}$ containing 5 mM EGTA and $10 \mathrm{~mm}$ ADVESEP-7 (Cydex, Lenexa, KS). Images were recorded on an inverted Nikon (Tokyo, Japan) TE2000 microscope equipped with epifluorescence and a $60 \times, 1.4$ numerical aperture objective, and FM4-64 dye uptake was quantified using MetaMorph integrated intensity function.

\section{Results}

\section{The unc-31 locus encodes the C. elegans homolog of CAPS}

The $u n c-31$ locus was originally isolated in a screen based on its locomotion defects and mapped to chromosome IV (Brenner, 1974). This region contains an open reading frame of $4062 \mathrm{nu}-$ cleotides encoding a 1353 aa protein with a molecular weight of $154 \mathrm{kDa}$ (Fig. 1A). The C. elegans UNC-31 protein is 51\% identical (70\% similar) to the human CAPS and 58\% identical $(74 \%$ similar) to D. melanogaster dCAPS. The domain structure of the UNC-31 protein (Fig. $1 B$ ) includes a pleckstrin homology $(\mathrm{PH})$ domain. The $\mathrm{PH}$ domain in rat CAPS has been shown to bind phosphatidylinositol-4, 5-biphosphate to facilitate membrane interactions and is essential for regulated dense-core vesicle exocytosis in PC12 cells (Grishanin et al., 2002). UNC-31 also contains a C2 domain of unknown function (Ann et al., 1997) and a MUN domain that occupies a large portion of the carboxy half of the protein (Fig. 1B). MUN domains were identified in Unc-13 (Basu et al., 2005) proteins and contain a syntaxin binding region essential for synaptic vesicle priming (Madison et al., 2005; Stevens et al., 2005).

To identify domains in CAPS/UNC-31 essential for function, we sequenced 26 existing or newly isolated $u n c-31$ alleles (Fig. $1 \mathrm{~A}$, Table 1). The $e 928$ allele contained a large deletion, and strains with this allele lacked CAPS/UNC-31 protein (Fig. 1 $A, C$ ), confirming that this is an $u n c-31$ null allele (Charlie et al., 2006). Many of the other mutant alleles consisted of frame shift, splicing, or nonsense mutations, and strains harboring these also lacked CAPS/UNC-31 protein (Fig. $1 A$, Table 1). Of the seven nonsense mutants sequenced, three (e375, e69, and u280) contained the same amber-suppressible mutation (Avery et al., 1993). mRNAs from mutants bearing the $u 377$ and sa534 alleles contained the same 60 bp deletion (Table 1 ) at the $3^{\prime}$ end of exon 5 and thus resulted in a 20 aa deletion. Nevertheless, these strains contained near wild-type levels of CAPS/UNC-31 protein (Fig. $1 C)$. Because these alleles resemble the nulls in phenotype, these data suggest that amino acids 273-293, which are conserved in vertebrate CAPS (50\% identical; $85 \%$ similar), correspond to a novel N-terminal region of the UNC-31 protein that is required for function (Fig. $1 B$ ). Of interest, this region of UNC-31 corresponds to a portion of a reported $\mathrm{p} 150^{\text {Glued }}$-binding domain in human CADPS2 (CAPS2) that is deleted in some autistic patients (Sadakata et al., 2007b).

Two mutants (ox299 and e714), which produce full-length CAPS/UNC-31 protein (Fig. 1C), contain missense mutations (Ile530Lys and Gly538Glu, respectively) in highly conserved residues in the C2 domain (Fig. 1D). Although ox299 only exhibits subtle defects in the worm, e714 exhibits a moderately strong defect in locomotion and nose tap response. For the latter, we cannot eliminate the possibility that increased degradation of the UNC-31 protein (Fig. 1C) contributes to the phenotype. However, functional studies of the cognate rat CAPS mutant proteins indicate that these mutations cause a severe loss-of-function as well as dominant-negative properties in secretion assays (Grishanin et al., 2004) (M.P. and T.F.J.M., unpublished observations). The data suggest that the C2 domain of UNC-31/CAPS plays an important functional role. In summary, these studies define an $\mathrm{N}$-terminal region of UNC-31/CAPS that may be part of a functional domain and suggest the functional importance of the $\mathrm{C} 2$ domain. In addition, mutant alleles confirm that the $u n c-31$ locus encodes the invertebrate homolog of CAPS.

\section{unc-31 mutants exhibit defects in locomotion and defecation} unc-31 mutants were isolated on the basis of defects in locomotion (Brenner, 1974) but were later shown to exhibit broad nervous system dysfunction extending to pharyngeal pumping, egglaying, and recovery from the dauer state (Avery et al., 1993). Similar defects have been observed in mutants affecting monoamines and neuropeptides (Nelson et al., 1998; Waggoner et al., 2000; Niacaris and Avery, 2003; Rogers et al., 2003; Chase et al., 2004; Dempsey et al., 2005). Because the role of monoamines and neuropeptides in locomotion is modulatory rather than essential, we considered whether unc-31 mutants exhibit defects in the modulation of movement rather than in the basic locomotor process. It was observed previously that well fed $u n c-31$ mutants are paralyzed but generate normal sinusoidal waves and move at near wild-type speeds when removed from food (Hodgkin et al., 1988; Avery et al., 1993). To investigate the movement defect in unc-31 mutants, we performed video tracking assays in the presence or absence of food. unc-31(e928) animals are nearly paralyzed on food, although older animals are slightly more active. We found that unc-31 (e928) animals that have just been removed from food were able to move at faster speeds (Fig. 2C). unc31(e928) animals food deprived for $5 \mathrm{~h}$ and assayed off food spent more time at higher speeds, approaching speeds achieved by the wild type on food. The ability of $u n c-31$ animals to move well off food indicates that UNC-31 is not essential for basal movement. The lack of movement on food is likely attributable to loss of a modulatory input, which would be consistent with dysfunction of neuropeptide or monoamine release in $u n c-31$ mutants.

The C. elegans defecation cycle is a stereotypic motor program that occurs approximately every $50 \mathrm{~s}$ (Thomas, 1990). This cycle begins with a posterior body contraction that forces gut contents to the anterior of the worm and is followed by an anterior body muscle contraction $(\mathrm{aBoc})$ that pushes gut contents toward the 


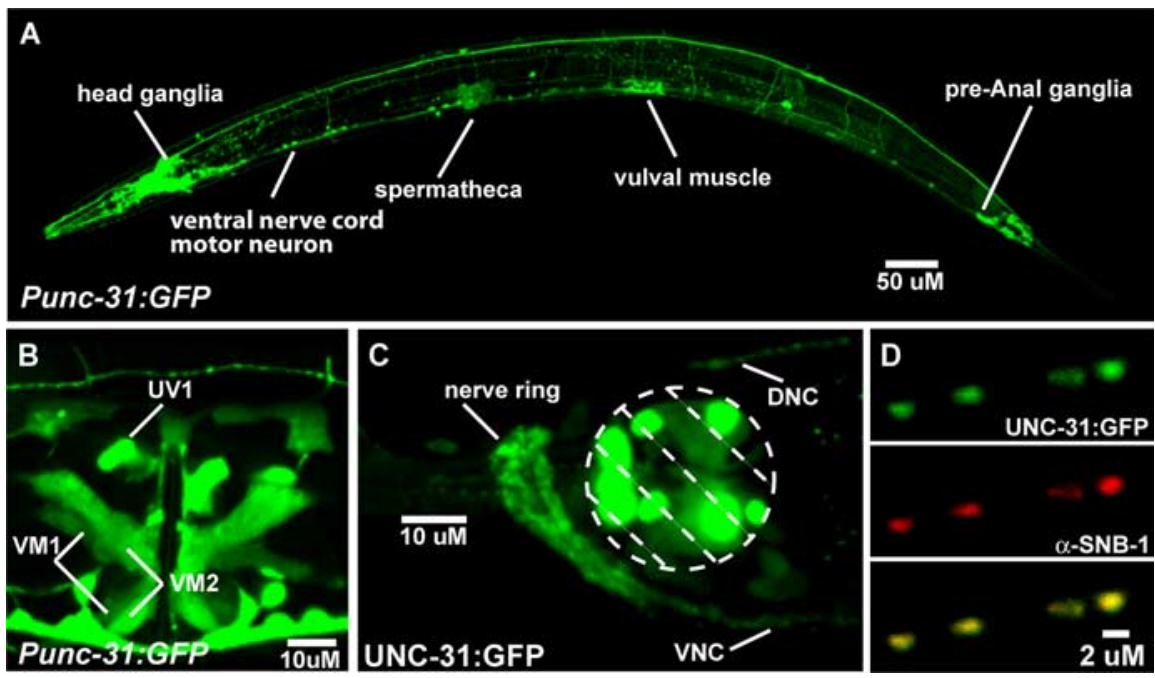

Figure 3. UNC-31 is expressed in neurons and is enriched at synapses. $\boldsymbol{A}$, Confocal image of an adult hermaphrodite expressing GFP under the unc-31 promoter (oxEx608[Punc-31:GFP]). Expression of unc-31 is pan-neuronal with additional expression in the spermatheca and vulval muscles. Anterior is left. $\boldsymbol{B}$, Confocal image of the ventral surface of an adult hermaphrodite expressing GFP under the unc-31 promoter. Expression is observed in the vulval muscles VM1 and VM2. Expression is also seen in the UV1 cells. C, Confocal images of unc-31(e928) oxEx608[UNC-31:GFP]. UNC-31:GFP protein localizes to the synaptic-rich region of the nerve ring as well as the dorsal and ventral nerve cords. GFP expression inside the dashed circle around the posterior bulb of the pharynx can be disregarded because it arises from the coinjection marker (Pmyo-2:HIS::GFP). Additional diffuse expression of the coinjection marker can also be seen in the isthmus of the pharynx. $D$, Subcellular localization of UNC-31:GFP in the SAB neurons of the head. Punctate expression as well as colocalization with the synaptic vesicle marker synaptobrevin ( $\alpha$-SNB-1) indicate that UNC-31 is localized to synaptic terminals, reported previously by Charlie et al. (2006).

posterior of the animal, which is followed by an enteric muscle contraction that expels gut contents (Emc). Two aspects of the defecation cycle were significantly disrupted in unc-31 mutants. First, unc-31 null alleles execute an anterior body contraction for only approximately half of the motor programs (Fig. $2 \mathrm{~A}$ ). Second, null mutants unc-31(e928) and unc-31(u280) displayed defects in cycle order (Fig. $2 \mathrm{~B}$ ) with the enteric muscle contraction preceding the anterior body contraction $\sim 75 \%$ of the time. Heterozygotes for the null mutations still displayed significant defects in the anterior body contraction but showed no defects in the order of the steps (Fig. $2 A, B$ ). This indicates that $u n c-31$ is haploinsufficient for the anterior body contraction step but not for cycle order. Thus, certain $u n c-31$ phenotypes are exquisitely sensitive to CAPS/UNC-31 protein levels. These defects are caused by mutations in $u n c-31$ because the UNC-31:GFP construct rescued the defecation (Fig. $2 A, B$ ) and movement defects (data not shown) of unc-31(e928) mutants.

\section{CAPS/UNC-31 is expressed in the nervous system}

Confocal analysis of strains containing the Punc-31::GFP transcriptional reporter revealed expression in the nerve ring, ventral nerve cord motor neurons, preanal ganglion, and head ganglion (Fig. 3A). There are 57 ventral nerve cord motor neurons and 12 preanal ganglion neurons (69 neurons) along the ventral side of the animal (WormAtlas). Counts of cell bodies in the ventral nerve cord and preanal ganglion revealed an average of $65 \pm 2$ $(n=4)$ GFP-positive cells, indicating that essentially all of the neurons of the ventral nerve cord and preanal ganglion express unc-31. Additional positive identifications were made for SDQ, PDE, BDU, ALM, DVA, DVB, DVC, HSN, and CAN neurons. Because these encompass most of the identifiable neurons in the background of a pan-neuronal expression pattern, we infer that unc-31 may be expressed in essentially all neurons. This is consistent with previous studies in D. melanogaster and mammals in which CAPS1 expression was restricted to the nervous and endocrine systems (Walent et al., 1992; Ann et al., 1997; Renden et al., 2001; Wassenberg and Martin, 2002). All reporter strains also displayed expression in the vulval muscles VM1 and VM2 (Fig. 3B) and occasional expression in what is likely VulE and VulF. Consistent expression was also noted in the UV1 cells (Fig. 3B) and the spermatheca (Fig. 3A). The UV1 cells are secretory and express other neuropeptides, monoamines, and other synaptic components (Nonet et al., 1993; Li et al., 1999; Zahn et al., 2001; Alkema et al., 2005). In summary, CAPS/UNC-31 is expressed throughout the nervous system and in other secretory cells.

\section{CAPS/UNC-31 localization in neurons}

Unlike synaptic vesicle release, dense-core vesicle release is not restricted to sites of synaptic contact. Components of densecore vesicles in $C$. elegans, including FMRFamide-related peptides, neuropeptideprocessing enzymes (Kass et al., 2001; Jacob and Kaplan, 2003), and IDA-1 (related to islet cell diabetes autoantigen-1) (Zahn et al., 2004), are localized throughout soma, axons, and synapses. We analyzed the localization of UNC-31 using a GFP fusion construct capable of rescuing the mutant phenotypes. UNC-31::GFP localized to neuronal cell bodies and axonal projections similarly to other dense-core vesicle components. Intense fluorescence was observed in regions containing synaptic contacts such as the nerve ring and the ventral and dorsal nerve cords (Fig. 3C). Because these areas are also dense with axonal projections, it was not possible to distinguish whether the UNC-31::GFP was present at synapses. To assess synaptic localization, we analyzed the presynaptic terminals of the SAB neurons of the head, which have relatively isolated synapses. Colocalization with synaptobrevin (SNB) indicated that UNC-31::GFP is present at sites of synaptic contact (Fig. 3D). Localization of UNC-31 to regions of synaptic contact has recently been demonstrated with antibody staining in C. elegans (Charlie et al., 2006).

\section{CAPS/UNC-31 is not required for neuronal development}

The widespread nervous system dysfunction displayed by $u n c-31$ null worms could indicate that UNC-31 plays a role in nervous system development. We examined neuronal cell differentiation, axon outgrowth, and synaptogenesis in $u n c-31$ null strains. To assess differentiation and axon outgrowth, a GFP marker that is expressed in the cell body and axons of GABA motor neurons was crossed into the unc-31(u280) background. The GABA motor nervous system consists of 26 cells with a subset of cells sending commissures across the midline to the DNC. Analysis revealed no defect in GABA motor neuron cell body number (Fig. 4C) or in the number of commissures (Fig. $4 A$ ) sent to the dorsal nerve cord in unc-31(u280) animals. No gross defects in axon projections were detected in this strain, indicating that pathfinding was normal. Furthermore, no defects in the density of synapses were detected by scoring synaptobrevin-GFP puncta in the GABA neuromuscular junctions of unc-31(u280) animals (Fig. 4B). 

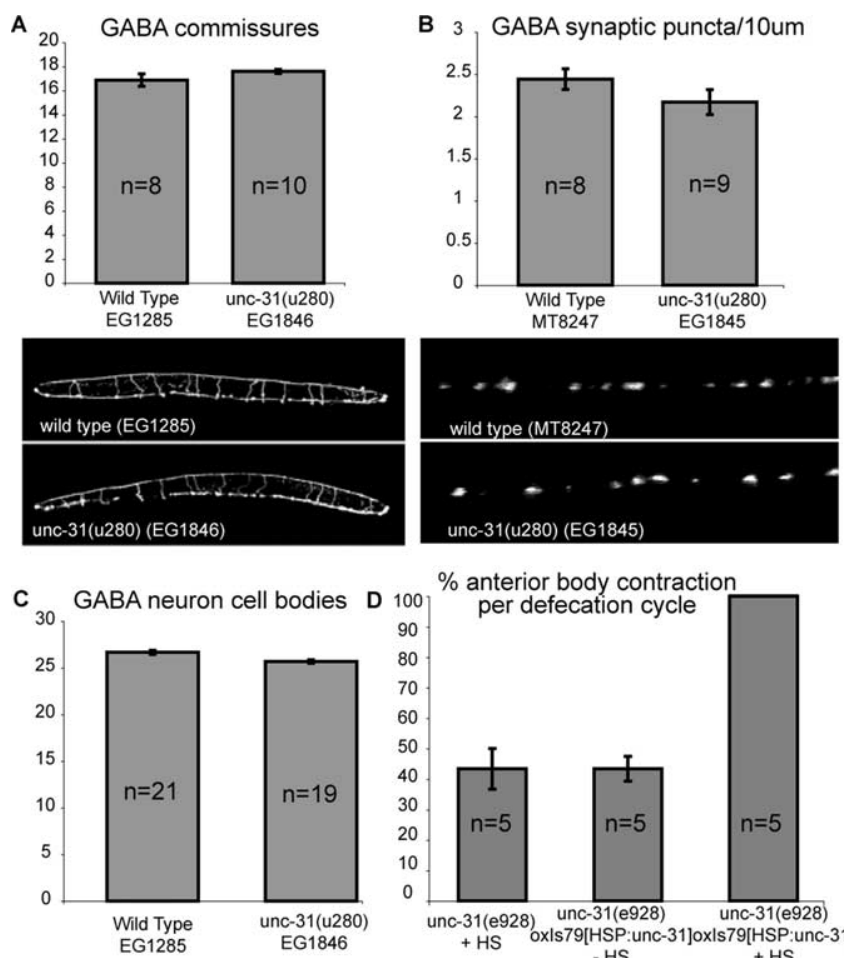

$\%$ anterior body contraction

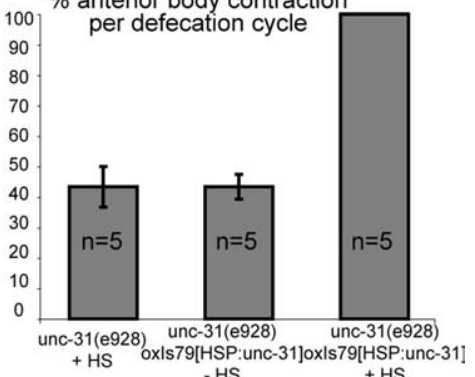

$\mathrm{HS}$

Figure 4. Absence of developmental defects in unc-31. A, Quantification of GABA motor neuron outgrowth indicates there are no defects in pathfinding in unc-31(u280). Confocal $Z$-series were captured of each animal at $40 \times$, and the numbers of axons that crossed the midline and terminated on the dorsal nerve cord were counted. $\boldsymbol{B}$, Synaptogenesis was evaluated by using GFP-tagged synaptobrevin (SNB-1:GFP). Quantification of SNB-1:GFP puncta density indicates that unc-31 mutants have normal numbers of GABA motor neuron synapses in the dorsal nerve cord. Puncta density was determined for a single animal by taking images of the dorsal nerve cord with a $63 \times$ lens at the posterior and anterior reflexes of the gonad. Puncta density in these two images was averaged to get the puncta per $10 \mu \mathrm{m}$. C, By counting the number of GFP-positive cells in a strain expressing GFP in GABA neurons (EG1846), it was determined that all GABA neurons are properly specified in unc-31 mutants. D, Acute expression of unc-37 from a heat shock (HS) promoter is able to rescue the anterior body contraction defect in unc-31(e928). Six cycles were observed for each animal scored to determine the percentage of cycles that had a contraction. All animals in these developmental assays were analyzed as young adults.

Development of the cholinergic nervous system was also normal in unc-31 mutants. Analysis of axon morphology and midline crossing in $u n c-31(u 280)$ animals expressing GFP in the acetylcholine motor neurons indicated there were no defects in axon pathfinding (data not shown). Synapse density in the dorsal nerve cord of cholinergic neurons in unc-31 (u280) animals (3.34 punc$\mathrm{ta} / 10 \mu \mathrm{m})$ was similar to that in wild-type animals (3.38 punc$\mathrm{ta} / 10 \mu \mathrm{m})$. Together, studies of the GABA and acetylcholine neurons indicated no defects in differentiation, axon outgrowth, or synaptogenesis in $u n c-31$ null mutants. This is consistent with the absence of gross developmental defects in CAPS mutants in mice and D. melanogaster (Renden et al., 2001; Speidel et al., 2005; Sadakata et al., 2007a).

To further rule out possible defects in neuronal development, we determined whether acute expression of UNC-31 would rescue phenotypic defects in mutant animals after the completion of nervous system development. Expression of UNC-31 using a heat shock promoter completely rescued defecation cycle defects in adult animals (Fig. 4D). These experiments are consistent with results demonstrating that a heat shock promoter-driven transgene containing a gain-of-function mutation in $\mathrm{G} \alpha_{\mathrm{s}}$ (Korswagen et al., 1997) strongly rescues the locomotion defects of unc-31

null mutants (Charlie et al., 2006). Thus, CAPS/UNC-31 is not required for development but instead is required for proper regulation of neuronally controlled behaviors in the mature animal.

\section{ANF-GFP is targeted to dense-core vesicles}

Dense-core vesicles are prevalent in C. elegans neurons in which at least 92 distinct neuropeptides are expressed (Li et al., 1999; Nathoo et al., 2001; Pierce et al., 2001), but there is no suitable functional assay for dense-core vesicle exocytosis in C. elegans. To directly assess the role of CAPS/UNC-31 in dense-core vesicle exocytosis, we established a novel assay using cultured embryonic neurons (Christensen et al., 2002) from C. elegans strains that express an ectopic neuropeptide, ANF-GFP.

The prodomain of a preproANF-GFP fusion protein directs the ectopically expressed neuropeptide to dense-core vesicles in PC12 cells (Burke et al., 1997) and in D. melanogaster (Shakiryanova et al., 2005, 2006) so that it can be used as a reliable fluorescent reporter of dense-core vesicle transport and exocytosis. We generated a C. elegans strain with an integrated extrachromosomal array that expresses preproANF-GFP under the control of the pan-neuronal aex-3 promoter. As anticipated, ANF-GFP was expressed throughout the nervous system in the integrated strain with fluorescence localized to the nerve ring as well as in the dorsal and ventral nerve cords (Fig. 5A). The ANF-GFP was concentrated in synaptic regions particularly in the nerve ring in which it colocalized with the synaptic proteins synaptotagmin and Rab3 (data not shown). The ANF-GFP was also distributed in neuronal cell bodies as would be anticipated for Golgi transport and dense-core vesicle packaging. ANF-GFP exhibited normal localization in unc-31 mutants (Fig. 5A).

ANF-GFP was properly targeted to dense-core vesicles in $C$. elegans based on two criteria. First, dense-core vesicle transport along axons can be observed using fluorescently tagged vesicle proteins (Zahn et al., 2004) and requires the kinesin UNC-104 for transport to the synapse (Jacob and Kaplan, 2003). Live imaging of ANF-GFP fluorescence in the nervous system revealed that GFP puncta moved along the length of commissural axons. Moreover, ANF-GFP was no longer present in synaptic regions but was mislocalized to cell bodies in unc-104 mutants (Fig. $5 A$ ). Second, ANF-GFP-containing vesicles exhibited a size distribution characteristic of dense-core vesicles. Postnuclear supernatants from homogenates of the ANF-GFP- and synaptogyrin (SNG)-GFP-expressing strains were analyzed by velocity sedimentation on sucrose gradients (Fig. $5 B$, top). Western blotting of gradient fractions with a monoclonal GFP antibody demonstrated that synaptogyrin-GFP (SNG-1:GFP, jsIs219), which is a synaptic vesicle component, sediments in fraction 14 (Fig. $5 B$, top). Synaptotagmin, which is localized to both synaptic vesicles and dense-core vesicles, exhibited peaks in fractions 14 with other synaptic vesicle components and with larger vesicles in fraction 10 (data not shown). Western blotting of gradient fractions from ANF-GFP-expressing strain (tgIs5[Paex-3:ANF::GFP]) revealed an expected $\sim 50 \mathrm{kDa}$ ANF-GFP fusion protein with a peak distribution in fractions $9-12$. Because $70 \mathrm{~nm}$ dense-core vesicles from PC12 cells sediment to fraction 6 in similar gradients (Martin and Kowalchyk, 1997), fractions 9-12 would be the expected location of the 37-52 nm dense-core vesicles in C. elegans (White et al., 1986). This was confirmed by finding that the mature form of the dense-core vesicle transmembrane IDA-1 protein was distributed in fractions 9-12 (Fig. 5B, bottom) (Zahn et al., 2004). Faster sedimenting material (fractions 2-7) in the gradient likely represents ANF-GFP in Golgi elements (Fig. 5B, top), which cosedimented with the uncleaved IDA-1 precursor (Fig. $5 B$, bot- 

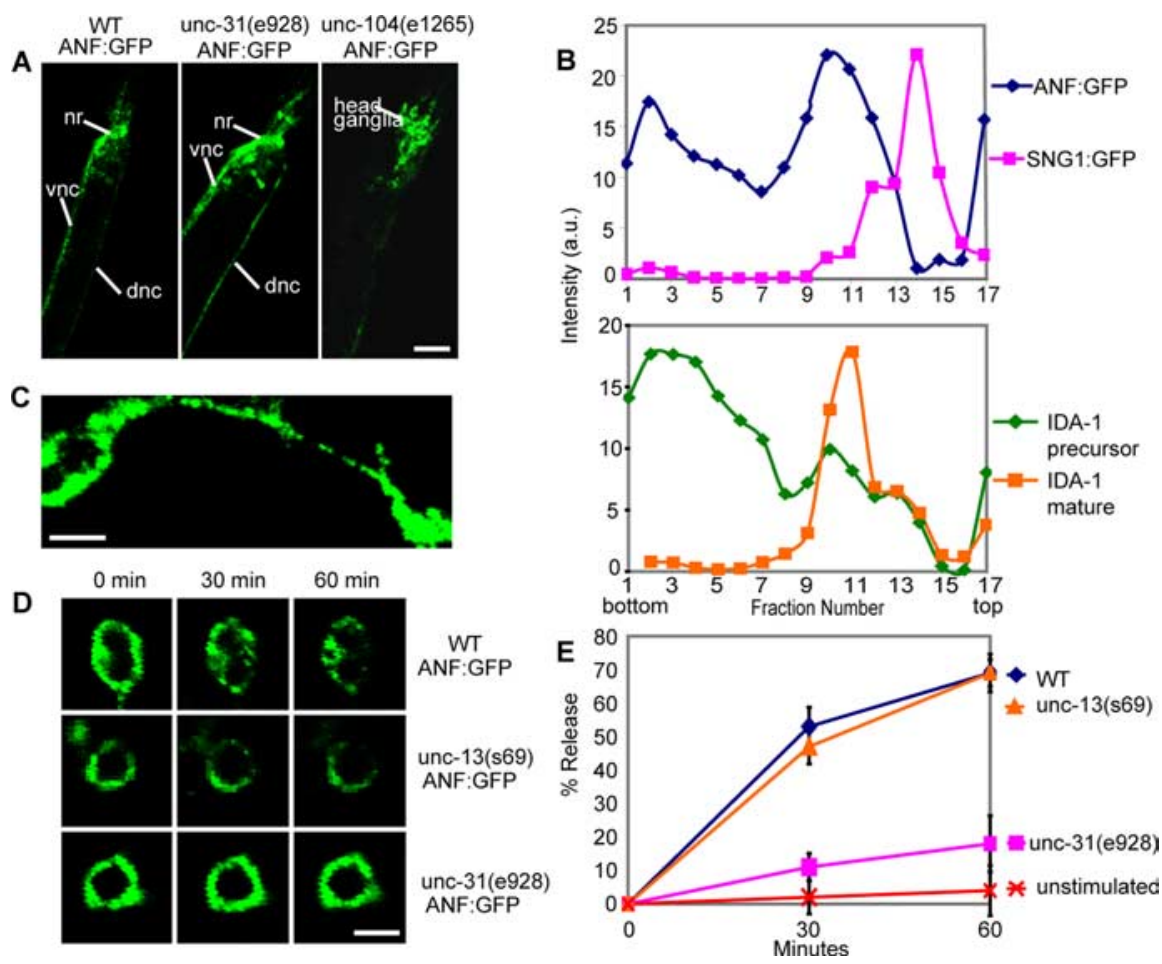

Figure 5. UNC-31 but not UNC-13 is essential for evoked dense-core vesicle exocytosis in cultured neurons. $A$, Images of the tgl55[Paex-3:ANF::GFP] strain in either wild-type (WT), unc-31(e928), or unc-104(e1265) backgrounds. Images are of adult hermaphrodites, and anterior is up. nr, Nerve ring; vnc, ventral nerve cord; dnc, dorsal nerve cord. Scale bar, $25 \mu \mathrm{m}$. B, Sucrose velocity sedimentation of $C$. elegans dense-core vesicles and synaptic vesicles. Dense-core vesicles and synaptic vesicles were prepared from tgls5[Paex-3:ANF::GFP] and js/s219[synaptogyrin::GFP] strains, respectively, and analyzed on sucrose gradients for corresponding ANF-GFP ( ) or synaptogyrin-GFP ( $\mathbf{\square})$ in the top. Western blotting was used to detect mature IDA-1 ( $\mathbf{\square}$ ) or IDA-1 precursor $(\checkmark)$ in the bottom. C, Image of a cultured neuron from the tgls5[Paex-3:ANF::GFP] strain in which the punctuate localization of ANF-GFP in cell bodies and neurites is shown. Scale bar, $2 \mu \mathrm{m}$. D, Time lapse images of ANF-GFP content in nerve cell bodies. Cultured neurons derived from tgls5 (wild type), tgls5 unc-31(e928), and tgls5 unc-13(s69) strains were imaged immediately after addition of stimulation buffer and at $30 \mathrm{~min}$ and $60 \mathrm{~min}$. E, Quantitation of ANF-GFP fluorescence in cultured neurons. Fluorescence in cell bodies was quantitated for wild-type neurons incubated in resting buffer $(n=14)(X)$ and in depolarization buffer for wild-type $(n=25)(\diamond)$, unc-37(e928) $(n=29)(\square)$, or unc-13(s69) $(n=24)(\mathbf{\Delta})$ neurons. Mean \pm SEM values of percentage release correspond to percentage fluorescence loss from cell bodies.

tom). These data indicate that ANF-GFP is localized to Golgi elements and is targeted to vesicular structures with the properties of dense-core vesicles.

To determine whether ANF-GFP is released from neurons in vivo, we quantitated the endocytic accumulation of ANF-GFP in coelomocyte cells. Coelomocytes are scavenger cells that continuously take up fluid from the pseudocoelomic space and would be expected to accumulate ANF-GFP that was released into the coelomic space. Indeed, ANF-GFP fluorescence was readily detected in coelomocytes in the wild-type strain. The overall expression level of ANF-GFP in the nervous system was comparable between the wild-type $u n c-31$ and $u n c-13$ mutant strains. However, lower levels of ANF-GFP fluorescence were observed in coelomocytes of both unc-31 mutant strains assayed (Fig. 6A,B). Most coelomocytes from wild-type animals accumulated high levels of ANF-GFP with fluorescence near saturation, whereas those from $u n c-31(e 928)$ or $u n c-31$ (u280) animals accumulated low levels of ANF-GFP and only rarely exhibited high fluorescence. We excluded the possibility that lack of ANF-GFP uptake by unc-31 (e928) coelomocytes was attributable to an endocytosis defect because coelomocytes in unc-31(e928) mutants were able to endocytose Texas Red-conjugated bovine serum albumin that was injected into the pseudocoelomic space.

To determine whether ANF:GFP release defects might arise generally from reduced levels of neuronal activity, we assayed coelomocyte ANF: GFP uptake in $u n c-13$ mutants that are deficient in synaptic vesicle fusion (Richmond et al., 1999). We found that unc13(s69) mutants displayed significant decreases in coelomocyte ANF:GFP levels as reported recently (Sieburth et al., 2007) but that unc-13(e51) mutants displayed wild-type coelomocyte ANF:GFP levels (Fig. 6A,B). These allele-specific effects are likely attributable to molecular differences in the two unc-13 alleles (Kohn et al., 2000), which disrupt synaptic activity to differing extents (Richmond et al., 1999). Although the coelomocyte ANF:GFP uptake results were consistent with defects in dense-core vesicle-mediated release in unc-31 mutants, they also indicated that decreased synaptic activity could also decrease ANF:GFP secretion. To circumvent the complication of synaptic activity effects on peptide secretion, we developed a cell culture assay for dense-core vesicle exocytosis.

\section{CAPS/UNC-31 but not UNC-13 is essential for regulated dense-core vesicle exocytosis}

To more directly assess the role of UNC-31 in activity-dependent dense-core vesicle exocytosis, we used cultured $C$. elegans neurons. Using the protocol established by Christensen et al. (2002) for culturing embryonic neurons, we developed a direct assay for the regulated release of ANF-GFP from dense-core vesicles. The primary cultured neurons exhibited ANFGFP in mobile vesicular structures in neurite extensions as well as in cell bodies (Fig. 5C,D). Incubations of the cells under resting conditions (without depolarization or divalent cations) over a 60 min period did not affect ANF-GFP fluorescence intensity. Depolarization in $\mathrm{Ba}^{2+}$-containing buffers effectively stimulates extensive neuropeptide secretion over an extended time period (Ng et al., 2002). We conducted incubations with depolarizing $\mathrm{Ba}^{2+}$-containing buffers (in mM: $86 \mathrm{KCl}$, $5 \mathrm{CaCl}_{2}$, and $5 \mathrm{BaCl}_{2}$ ), which led to the extensive $(\sim 70 \%)$ reduction in ANF-GFP fluorescence from cell bodies of the neurons (Fig. $5 D, E$ ) and the appearance of ANF-GFP fluorescence in the extracellular buffer (data not shown). To determine the function of CAPS/UNC-31 and other proteins in dense-core vesicle exocytosis, we crossed the ANF::GFP integrated strain with mutant strains to generate cultured neurons expressing ANF-GFP in different genetic backgrounds.

UNC-13 has a well characterized role in the priming of synaptic vesicles for exocytosis (Richmond et al., 1999, 2001). However, the role of UNC-13 in neuronal dense-core vesicle exocytosis has not been established. To assess this function for UNC-13, we measured evoked ANF-GFP release from cultured neurons derived from $C$. elegans harboring a strong loss-of-function unc-13(s69) mutation. There was no detectable defect in stimulated ANF-GFP release (Fig. $5 D, E$ ), which indicates that UNC-13 does not play an essential role in neuronal dense-core vesicle exocytosis. 
Similar assays conducted with cultured neurons derived from $C$. elegans harboring a null allele of $u n c-31(e 928)$ revealed that evoked ANF-GFP release was nearly abolished and was indistinguishable from release observed in unstimulated wildtype neurons (Fig. 5D,E). unc-31(e928) neurons exhibited a normal subcellular distribution of vesicular ANF-GFP, indicating that dense-core vesicle synthesis was normal in unc-31(e928) neurons. The results indicate that evoked dense-core vesicle exocytosis requires CAPS/UNC-31.

\section{UNC-13 but not CAPS/UNC-31 is required for regulated synaptic vesicle recycling}

The cultured neuron preparation also provided the opportunity to directly assess the role of CAPS/UNC-31 in regulated synaptic vesicle exocytosis by conducting assays for synaptic vesicle recycling. Synaptic vesicles in synaptic terminals undergo stimulated exocytosis and compensatory endocytosis, which can be detected as the stimulated uptake of a membrane-binding styryl dye such as FM4-64 (Betz and Bewick, 1992). Cultured neurons expressing GFP-tagged synaptogyrin ( jsIs219) were used to identify synaptic terminals (Nonet, 1999). Synaptic uptake of FM4-64 was readily detected in cultures incubated in depolarizing buffers with calcium $(56 \mathrm{mM} \mathrm{KCl}$ and 5 $\mathrm{mM} \mathrm{CaCl}_{2}$ ) (Fig. $7 A$, top row). In contrast, FM4-64 uptake was not observed for incubations in buffers lacking depolarizing $\mathrm{K}^{+}$ or $\mathrm{Ca}^{2+}$ (Fig. 7A, rows 3 and 5). Thus, FM4-64 dye loading by cultured C. elegans neurons was $\mathrm{Ca}^{2+}$ and depolarization dependent as expected for stimulated synaptic vesicle recycling.

Synaptic transmission is essentially abolished in the motor neurons of unc13(s69) mutants (Richmond et al., 1999), indicating a virtually complete failure of synaptic vesicle exocytosis. Consistent with this, neurons from unc-13(s69) worms exhibited no stimulated uptake of FM4-64 (Fig. 7A, row 4, $B$ ). This result provides an important control for the specificity of FM4-64 uptake for synaptic vesicle recycling in the culture system and independently confirms the essential role of UNC-13 in synaptic vesicle exocytosis (Aravamudan et al., 1999; Augustin et al., 1999; Richmond et al., 1999). In marked contrast, the stimulated uptake of FM4-64 into neurons from the unc-31(e928) strain was indistinguishable from that of wild-type neurons (Fig. 7A, row 2, $B)$. Because the synapses in primary culture display heterogeneity with variation in FM4-64 fluorescence loading, we binned the data to derive a profile of loaded synapse intensities (Fig. 7C). Unstimulated wild-type and stimulated unc-13 neurons displayed the same intensity distribution, whereas stimulated $u n c-31$ neurons displayed an intensity distribution that was indistinguishable from that of
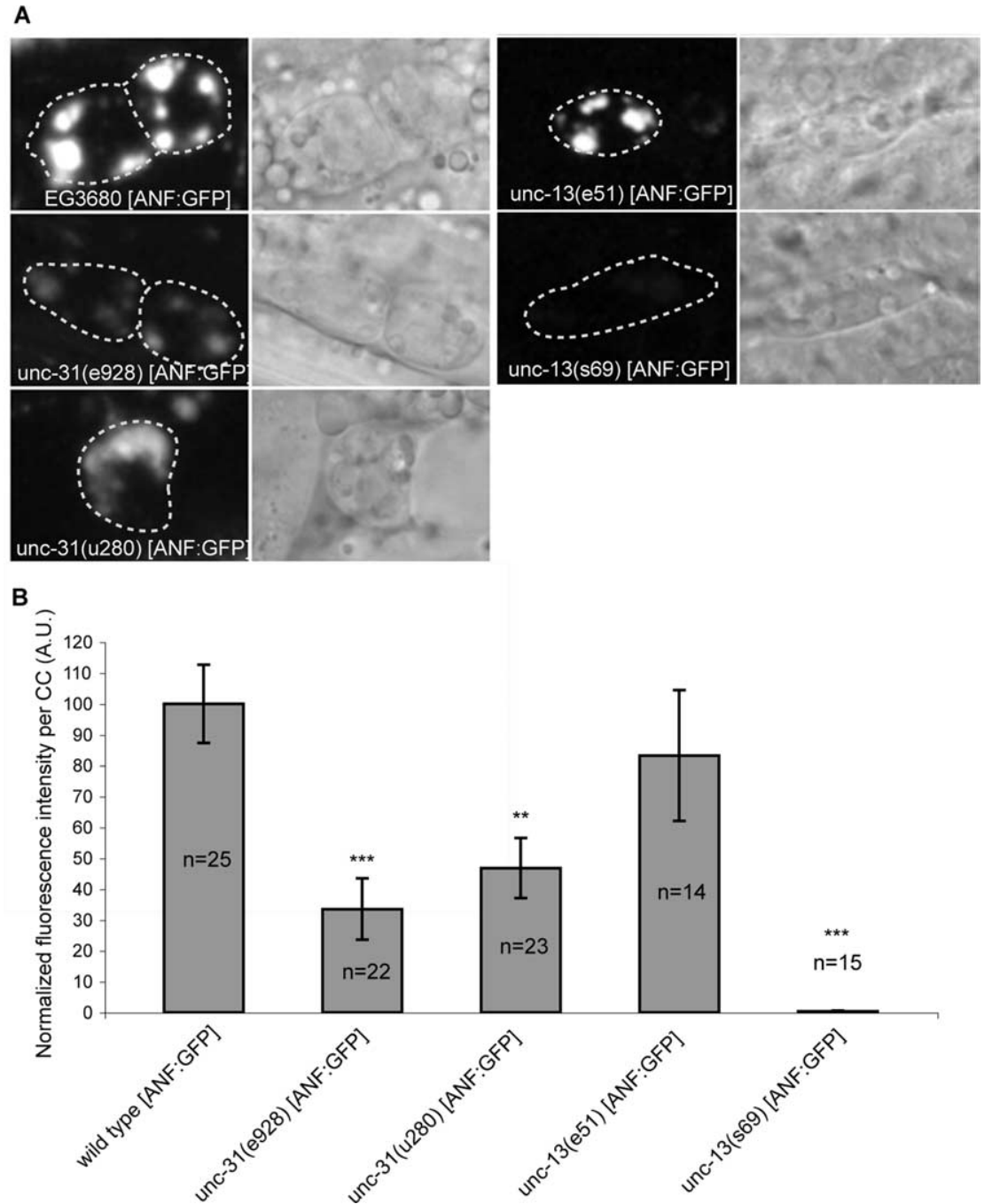

Figure 6. unc-31 mutants release less ANF-GFP into the body cavity. $\boldsymbol{A}$, For each strain, the left represents a maximum pixel intensity projection of the GFP signal arising from ANF-GFP that has been taken up into endocytic compartments of the coelomocytes. The yellow dashed line indicates the location of the coelomocytes, which are shown in DIC images on the right. Fluorescence outside of the yellow dashed line is gut autofluorescence. $\boldsymbol{B}$, Graph showing the normalized total pixel intensity per coelomocyte (A.U.) of 100 to enable comparison of separate experiments in which the wild-type strain EG3680 ox/s206[ANF:GFP] was compared with mutant strains EG3681 unc-31(e928) oxls206[ANF:GFP], EG3682 unc-31(u280) ox/s206[ANF:GFP]), EG3345 unc-13(s69) brate output of the microscope for fluorescence normalization. The SEM for the control strain (EG3680) was calculated as a weighted (based on total $n$ ) average of the SEM from three separate experiments, which differed by $<8 \%$. Statistical analyses were conducted for data within an experiment, and all genotypes were singly compared with control via an unpaired $t$ test $\left({ }^{* *} p=\right.$ $\left.0.002,{ }^{* * *} p<0.0001\right)$. The complete lack of ANF:GFP in coelomocytes of unc-13(s69) mutants is only reflective of the analyzed posterior coelomocytes, whereas anterior coelomocytes contained some ANF:GFP.

stimulated wild-type neurons. The combined data from cultured neurons indicate that CAPS/UNC-31 is essential for evoked densecore vesicle but not synaptic vesicle recycling. Conversely, UNC-13 is essential for evoked synaptic vesicle but not dense-core vesicle exocytosis.

\section{Discussion}

Our results lead to four major conclusions. First, the unc-31 gene in C. elegans encodes a CAPS protein that is very similar to vertebrate orthologs and is expressed in most if not all neurons and in a few secretory cells outside the nervous system in C. elegans. Second, the loss-of-function unc-31 phenotype, which includes widespread nervous system dysfunction, does not arise from de- 
FM4-64

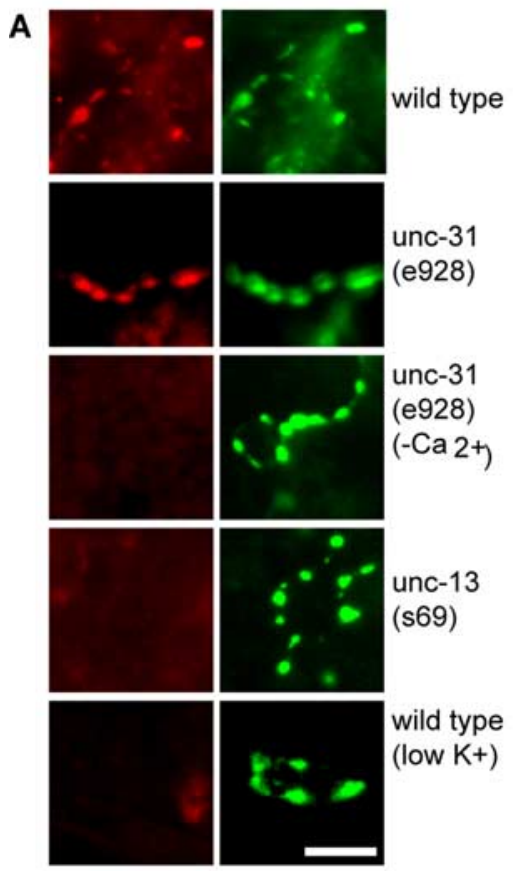

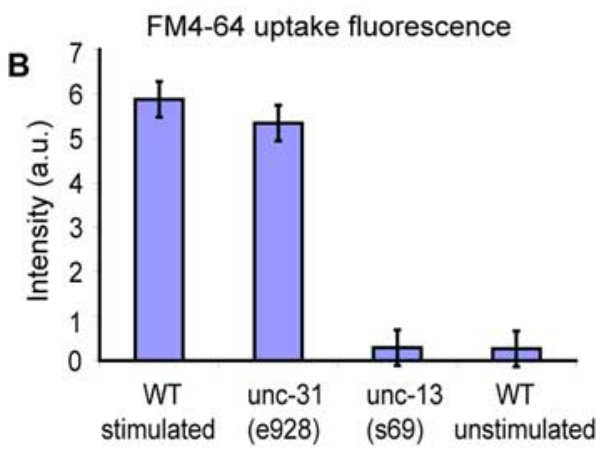

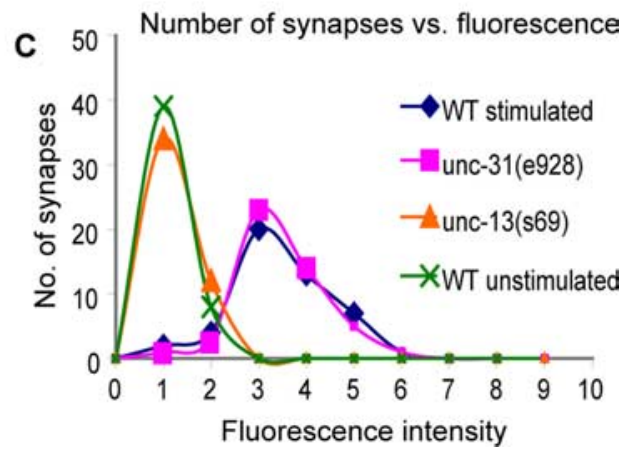

Figure 7. UNC-13 but not UNC-31 is essential for evoked synaptic vesicle exocytosis in cultured neurons. $\boldsymbol{A}$, Cultured neurons were prepared from js/s219 [synaptogyrin::GFP] strains of different genotypes. FM4-64 dye uptake was stimulated in $\mathrm{K}^{+}$depolarization buffer for wild-type or unc-31(e928) or unc-13(s69) neurons as indicated. Left column corresponds to FM4-64 fluorescence, and right column corresponds to synaptogyrin-GFP in terminals. Bottom two rows correspond to control studies (high $\mathrm{K}^{+}$ buffer without $\mathrm{CaCl}_{2}$ or low $\mathrm{K}^{+}$buffer) in wild-type or unc-31 neurons, indicating that FM4-64 uptake fails to occur under control conditions. Scale bar, $2 \mu \mathrm{m}$. B, Quantitation of FM4-64 uptake in wild-type (WT), unc-31(e928), and unc-13(s69) neurons in depolarization buffer and in wild-type neurons in resting buffer (control). For each condition, $n=50$. Mean \pm SEM values are shown. C, Binned data of FM4-64 uptake. Intensity distributions are shown for unstimulated wild-type neurons $(X)$ and stimulated unc-13(s69) ( $\mathbf{\Delta})$, wild-type ( $\bullet$ ), or unc-31(e928) ( $\square$ ) neurons.

velopmental defects. Third, UNC-31 function is required for dense-core vesicle exocytosis but not synaptic vesicle recycling in the nervous system of C. elegans. Fourth, UNC-13, which shares sequence homology with CAPS/UNC-31 and is essential for synaptic vesicle exocytosis, is not required for neuronal dense-core vesicle exocytosis. Together, our results suggest that CAPS/ UNC-31 and UNC-13 are functional homologs that operate selectively in dense-core vesicle and synaptic vesicle exocytosis, respectively, in the nervous system of C. elegans.

Vertebrate CAPS1 is exclusively expressed in neurons and peptide hormone-secreting endocrine cells (Walent et al., 1992; Ann et al., 1997; Wassenberg and Martin, 2002), whereas vertebrate CAPS2 is expressed throughout the nervous system but also widely in other secretory tissues (Cisternas et al., 2003; Speidel et al., 2003; Sadakata et al., 2007b). Our analysis of a transcriptional GFP reporter indicated that UNC-31 is expressed in most neurons of the hermaphrodite. Recent immunocytochemical studies (Charlie et al., 2006) indicated a more restricted distribution of UNC-31 protein in cholinergic and some noncholinergic synapses, but this may reflect different sensitivities of the techniques used. We also observed expression of UNC-31 in the UV1 uterine secretory cells, which is consistent with previous findings that these cells express IDA-1, a homolog of the dense-core vesicle membrane protein phogrin, as well as FMRFamide-like peptides, and tyramine (Li et al., 1999; Zahn et al., 2001; Kim and Li, 2004; Alkema et al., 2005). UNC-31 expression was also detected in the vulval muscles, which have been shown to express the FMRFamide neuropeptide FLP-10 (FMRF-like peptide-10) (Kim and Li,
2004). These results are consistent with a function for UNC-31 in dense-core vesicle release.

Previous characterization of the $u n c-31$ mutant phenotype in C. elegans identified multiple aspects of nervous system dysfunction beyond the uncoordinated locomotion phenotype. These included constitutive pharyngeal pumping, defective egg-laying, reduced recovery from the dauer state (Avery et al., 1993), defects in defecation (Miller et al., 1996), and prolonged lifespan (Ailion et al., 1999). Our studies indicated that the unc-31 movement defects do not result from defects in neuronal differentiation, axon outgrowth, or synaptogenesis. Moreover, the acute rescue of defecation cycle defects by heat shock-induced expression of UNC-31 indicated that the mutant phenotype arises from defects in neuron function in the mature animal rather than developmental defects. A similar lack of involvement in neural development was found in $d C A P S$ nulls in D. melanogaster (Renden et al., 2001) and in CAPS $1^{-/-} /$CAPS $2^{+/+}$mice (Speidel et al., 2005), whereas deletion of CAPS2 from mice caused developmental alterations in the cerebellum attributable to deficiencies in neurotrophin secretion (Sadakata et al., 2007a).

Studies of CAPS1 function in vertebrate cells indicate that CAPS1 is required for regulated dense-core vesicle exocytosis (Ann et al., 1997; Berwin et al., 1998; Tandon et al., 1998; Elhamdani et al., 1999; Rupnik et al., 2000; Grishanin et al., 2002, 2004; Speidel et al., 2003, 2005), but genetic studies to assess CAPS1 function are complicated by redundancy with CAPS2. We assessed the requirement for CAPS/UNC-31 in dense-core vesicle release in $C$. elegans by targeting ANF-GFP to dense-core vesicles. This ANF-GFP reporter has been used to study dense-core vesicle transport and exocytosis in vertebrate and invertebrate cells (Burke et al., 1997; Ng et al., 2002; Shakiryanova et al., 2005, 2006). Transgenic worms expressing ANFGFP in neurons packaged this protein into organelles with the properties of dense-core vesicles. In wild-type worms, ANF-GFP was secreted into the pseudocoelomic space as monitored by its accumulation by coelomocytes, whereas little ANF-GFP secretion was detected in unc-31 mutants. In contrast, unc-13(e51) mutants exhibited wild-type characteristics, whereas unc-13(s69) mutants exhibited decreased ANF:GFP secretion in this in vivo assay. Previous molecular characterization of these two unc-13 alleles revealed that $u n c-13$ (s69) disrupts both left, right (LR) and middle, right transcripts, whereas unc-13(e51) only disrupts the LR transcript (Kohn et al., 2000). These two alleles display different electrophysiological phenotypes, with unc-13(s69) exhibiting essentially no transmitter release whereas some release persists in unc-13(e51) mutants (Richmond et al., 1999). We interpret the effect of unc-13(s69) on coelomocyte ANF:GFP uptake to indicate that synaptic activity can regulate peptide secretion. To more directly assess the role of UNC-31 in dense-core vesicle exocytosis, we used an isolated cell culture system. Depolarization of cultured neurons from worms carrying an integrated ANF:GFP 
array in $\mathrm{Ba}^{2+}$ elicited extensive release of ANF-GFP, whereas unc-31(e928) worms were deficient for the evoked release of dense-core vesicle contents. From these results, we conclude that regulated dense-core vesicle exocytosis is defective in unc-31 mutants.

Consistent with this conclusion, studies in P30 adrenal chromaffin cells derived from a heterozygous $\mathrm{CAPS}^{-/+}$mouse, which lack CAPS2, showed that a $\sim 35 \%$ reduction in CAPS 1 was associated with a $30-35 \%$ reduction in the readily releasable dense-core vesicle pool (Speidel et al., 2005). However, embryonic day 18 adrenal chromaffin cells derived from a homozygous $\mathrm{CAPS1}^{-/-}$mouse, which contain CAPS2, exhibited normal exocytosis of dense-core vesicles (Speidel et al., 2005). Although this was likely attributable to CAPS2 expression and functional redundancy, the authors (Speidel et al., 2005) also reported defects in catecholamine secretion and concluded that CAPS1 was essential for proper catecholamine uptake or retention by dense-core vesicles. Catecholamine loading or storage is unlikely to be a primary function for UNC-31 in C. elegans. First, null mutants in the $C$. elegans vesicular monoamine transporter (VMAT), encoded by cat-1 (abnormal catecholamine distribution family member) are not paralyzed on food like unc-31 (Duerr et al., 1999). Second, VMAT and catecholamines (dopamine, serotonin, octopamine, and tyramine) are restricted to a small $(\sim 30)$ subset of neurons in C. elegans (Sulston et al., 1975; Horvitz et al., 1982; Desai et al., 1988; Sawin, 1996; Rand and Nonet 1997; Duerr et al., 1999; Alkema et al., 2005), whereas UNC-31 is expressed in many if not all neurons. Third, the movement defects in an unc-31 null mutant can be rescued to near wild-type levels by expressing an $u n c-31$ transgene solely in a subset of the ventral cord cholinergic neurons (Charlie et al., 2006) that do not express catecholamines or VMAT (Sulston et al., 1975; Horvitz et al., 1982; Desai et al., 1988; Duerr et al., 1999; Alkema et al., 2005). Fourth, as shown here, $u n c-31$ mutants exhibit profound defects in evoked peptide secretion, indicating that CAPS/UNC-31 functions broadly in dense-core vesicle exocytosis and is not restricted to catecholamine secretion.

CAPS/UNC-31 shares a large domain with the Unc13 family of proteins called the MUN domain. C. elegans UNC-13, Drosophila Dunc13, and the vertebrate Munc-13 are essential for a prefusion priming step in synaptic vesicle exocytosis (Aravamudan et al., 1999; Augustin et al., 1999; Richmond et al., 1999). This sequence homology and the fact that CAPS mutants in C. elegans (Miller et al., 1996) and D. melanogaster (Renden et al., 2001) display mild defects in synaptic transmission in vivo could indicate that CAPS also functions in the exocytosis of synaptic vesicles. Alternatively, mild deficiencies in synaptic transmission in vivo could be caused by defects in modulatory transmitter secretion as was suggested for the non-cell-autonomous function of dCAPS in D. melanogaster (Renden et al., 2001). To directly address the possible role of CAPS/UNC-31 in synaptic vesicle exocytosis, we used cultured neurons that are free of neuronal circuitry and diffusible mediators. We found that stimulationdependent synaptic uptake of FM4-64 in unc-31(928) cultured neurons was indistinguishable from that of wild-type neurons. These results indicate that UNC-31 is not required for evoked synaptic vesicle recycling, although we cannot eliminate the possibility that a small subset of synaptic vesicles was not detected in the FM4-64 dye-loading studies. In contrast, neurons from unc13(s69) mutants were entirely deficient for stimulated FM4-64 uptake as would be anticipated from previous results documenting strong deficiencies in synaptic transmission (Aravamudan et al., 1999; Augustin et al., 1999; Richmond et al., 1999). Overall, the evidence indicates that CAPS/UNC-31 does not play a direct role in synaptic vesicle exocytosis at least in the dominant population of cholinergic and glutamatergic neurons present in cultures of C. elegans neurons.

Although CAPS/UNC-31 functioned selectively in dense-core vesicle exocytosis, the possibility exists that UNC-13 might also function in this pathway. However, we found that evoked ANFGFP release in $u n c-13$ (s69) neurons was indistinguishable from that in wild-type neurons. Thus, despite the sequence homology to CAPS/UNC-31, UNC-13 did not appear to be essential for dense-core vesicle release in cultured $C$. elegans neurons. This finding is consistent with the observation that evoked dense-core vesicle exocytosis is unaltered in chromaffin cells from Munc13-1 knock-out mice (Stevens et al., 2005) but contradictory to reported deficits in stimulated insulin secretion from pancreatic $\beta$ cells from the same mice (Kang et al., 2006; Kwan et al., 2006). This may indicate that the multiple CAPS and Munc13 isoforms in vertebrates function in parallel in some secretory processes. Overall, our results suggest a nonoverlapping functional complementarity between UNC-31 and UNC-13 for dense-core vesicle and synaptic vesicle exocytosis, respectively, in the C. elegans nervous system.

\section{References}

Ailion M, Inoue T, Weaver CI, Holdcraft RW, Thomas JH (1999) Neurosecretory control of aging in Caenorhabditis elegans. Proc Natl Acad Sci USA 96:7394-7397.

Alkema MJ, Hunter-Ensor M, Ringstad N, Horvitz HR (2005) Tyramine functions independently of octopamine in the Caenorhabditis elegans nervous system. Neuron 46:247-260.

Ann K, Kowalchyk JA, Loyet KM, Martin TF (1997) Novel Ca ${ }^{2+}$-binding protein (CAPS) related to UNC-31 required for $\mathrm{Ca}^{2+}$-activated exocytosis. J Biol Chem 272:19637-19640.

Aravamudan B, Fergestad T, Davis WS, Rodesch CK, Broadie K (1999) Drosophila UNC-13 is essential for synaptic transmission. Nat Neurosci 2:965-971.

Augustin I, Rosenmund C, Sudhof TC, Brose N (1999) Munc13-1 is essential for fusion competence of glutamatergic synaptic vesicles. Nature 400:457-461.

Avery L, Bargmann CI, Horvitz HR (1993) The Caenorhabditis elegans unc-31 gene affects multiple nervous system-controlled functions. Genetics 134:455-464.

Basu J, Shen N, Dulubova I, Lu J, Guan R, Guryev O, Grishin NV, Rosenmund C, Rizo J (2005) A minimal domain responsible for Munc13 activity. Nat Struct Mol Biol 12:1017-1018.

Berwin B, Floor E, Martin TF (1998) CAPS (mammalian UNC-31) protein localizes to membranes involved in dense-core vesicle exocytosis. Neuron 21:137-145.

Betz WJ, Bewick GS (1992) Optical analysis of synaptic vesicle recycling at the frog neuromuscular junction. Science 255:200-203.

Brenner S (1974) The genetics of Caenorhabditis elegans. Genetics 77:71-94. Burke NV, Han W, Li D, Takimoto K, Watkins SC, Levitan ES (1997) Neuronal peptide release is limited by secretory granule mobility. Neuron 19:1095-1102.

Charlie NK, Schade MA, Thomure AM, Miller KG (2006) Presynaptic UNC-31 (CAPS) is required to activate the G alpha(s) pathway of the Caenorhabditis elegans synaptic signaling network. Genetics 172:943-961.

Chase DL, Pepper JS, Koelle MR (2004) Mechanism of extrasynaptic dopamine signaling in Caenorhabditis elegans. Nat Neurosci 7:1096-1103.

Christensen M, Estevez A, Yin X, Fox R, Morrison R, McDonnell M, Gleason C, Miller III DM, Strange K (2002) A primary culture system for functional analysis of C. elegans neurons and muscle cells. Neuron 33:503-514.

Cisternas FA, Vincent JB, Scherer SW, Ray PN (2003) Cloning and characterization of human CADPS and CADPS2, new members of the $\mathrm{Ca}^{2+}$ dependent activator for secretion protein family. Genomics 81:279-291.

Dempsey CM, Mackenzie SM, Gargus A, Blanco G, Sze JY (2005) Serotonin (5HT), fluoxetine, imipramine and dopamine target distinct 5HT receptor signaling to modulate Caenorhabditis elegans egg-laying behavior. Genetics 169:1425-1436. 
Desai C, Garriga G, McIntire SL, Horvitz HR (1988) A genetic pathway for the development of the Caenorhabditis elegans HSN motor neurons. Nature 336:638-646.

Duerr JS, Frisby DL, Gaskin J, Duke A, Asermely K, Huddleston D, Eiden LE, Rand JB (1999) The cat-1 gene of Caenorhabditis elegans encodes a vesicular monoamine transporter required for specific monoaminedependent behaviors. J Neurosci 19:72-84.

Elhamdani A, Martin TF, Kowalchyk JA, Artalejo CR (1999) $\mathrm{Ca}^{2+}$ dependent activator protein for secretion is critical for the fusion of dense-core vesicles with the membrane in calf adrenal chromaffin cells. J Neurosci 19:7375-7383.

Grishanin RN, Klenchin VA, Loyet KM, Kowalchyk JA, Ann K, Martin TF (2002) Membrane association domains in $\mathrm{Ca}^{2+}$-dependent activator protein for secretion mediate plasma membrane and dense-core vesicle binding required for $\mathrm{Ca}^{2+}$-dependent exocytosis. J Biol Chem 277:22025-22034.

Grishanin RN, Kowalchyk JA, Klenchin VA, Ann K, Earles CA, Chapman ER, Gerona RR, Martin TF (2004) CAPS acts at a prefusion step in densecore vesicle exocytosis as a $\mathrm{PIP}_{2}$ binding protein. Neuron 43:551-562.

Hodgkin J, Edgeley M, Riddle DL, Albertson DG (1988) Appendix 4: genetics. In: The nematode Caenorhabditis elegans (Wood W, Community of C. elegans Researchers, eds), pp 491-584. Cold Spring Harbor, NY: Cold Spring Harbor Laboratory.

Horvitz HR, Chalfie M, Trent C, Sulston JE, Evans PD (1982) Serotonin and octopamine in the nematode Caenorhabditis elegans. Science 216:1012-1014.

Jacob TC, Kaplan JM (2003) The EGL-21 carboxypeptidase E facilitates acetylcholine release at Caenorhabditis elegans neuromuscular junctions. J Neurosci 23:2122-2130.

Jahn R, Lang T, Sudhof TC (2003) Membrane fusion. Cell 112:519-533.

Kang L, He Z, Xu P, Fan J, Betz A, Brose N, Xu T (2006) Munc13-1 is required for the sustained release of insulin from pancreatic beta cells. Cell Metab 3:463-468.

Kass J, Jacob TC, Kim P, Kaplan JM (2001) The EGL-3 proprotein convertase regulates mechanosensory responses of Caenorhabditis elegans. J Neurosci 21:9265-9272.

Kim K, Li C (2004) Expression and regulation of an FMRFamide-related neuropeptide gene family in Caenorhabditis elegans. J Comp Neurol 475:540-550.

Kohn RE, Duerr JS, McManus JR, Duke A, Rakow TL, Maruyama H, Moulder G, Maruyama IN, Barstead RJ, Rand JB (2000) Expression of multiple UNC-13 proteins in the Caenorhabditis elegans nervous system. Mol Biol Cell 11:3441-3452.

Korswagen HC, Park JH, Ohshima Y, Plasterk RH (1997) An activating mutation in a Caenorhabditis elegans Gs protein induces neural degeneration. Genes Dev 11:1493-1503.

Kwan EP, Xie L, Sheu L, Nolan CJ, Prentki M, Betz A, Brose N, Gaisano HY (2006) Munc13-1 deficiency reduces insulin secretion and causes abnormal glucose tolerance. Diabetes 55:1421-1429.

Li C, Kim K, Nelson LS (1999) FMRFamide-related neuropeptide gene family in Caenorhabditis elegans. Brain Res 848:26-34.

Livingstone D (1991) Studies on the unc-31 gene of Caenorhabditis elegans. PhD thesis, University of Cambridge.

Madison JM, Nurrish S, Kaplan JM (2005) UNC-13 interaction with syntaxin is required for synaptic transmission. Curr Biol 15:2236-2242.

Martin TF (2003) Tuning exocytosis for speed: fast and slow modes. Biochim Biophys Acta 1641:157-165.

Martin TF, Kowalchyk JA (1997) Docked secretory vesicles undergo $\mathrm{Ca}^{2+}$. activated exocytosis in a cell-free system. J Biol Chem 272:14447-14453.

Martin TF, Walent JH (1989) A new method for cell permeabilization reveals a cytosolic protein requirement for $\mathrm{Ca}^{2+}$-activated secretion in GH3 pituitary cells. J Biol Chem 264:10299-10308.

Mello CC, Kramer JM, Stinchcomb D, Ambros V (1991) Efficient gene transfer in C. elegans: extrachromosomal maintenance and integration of transforming sequences. EMBO J 10:3959-3970.

Miller KG, Alfonso A, Nguyen M, Crowell JA, Johnson CD, Rand JB (1996) A genetic selection for Caenorhabditis elegans synaptic transmission mutants. Proc Natl Acad Sci USA 93:12593-12598.

Nathoo AN, Moeller RA, Westlund BA, Hart AC (2001) Identification of neuropeptide-like protein gene families in Caenorhabditis elegans and other species. Proc Natl Acad Sci USA 98:14000-14005.

Nelson LS, Rosoff ML, Li C (1998) Disruption of a neuropeptide gene, flp-1, causes multiple behavioral defects in Caenorhabditis elegans. Science 281:1686-1690.

Ng YK, Lu X, Levitan ES (2002) Physical mobilization of secretory vesicles facilitates neuropeptide release by growth factor-differentiated PC12 cells. J Physiol (Lond) 542:395-402.

Niacaris T, Avery L (2003) Serotonin regulates repolarization of the C. elegans pharyngeal muscle. J Exp Biol 206:223-231.

Nonet ML (1999) Visualization of synaptic specializations in live C. elegans with synaptic vesicle protein-GFP fusions. J Neurosci Methods 89:33-40.

Nonet ML, Grundahl K, Meyer BJ, Rand JB (1993) Synaptic function is impaired but not eliminated in C. elegans mutants lacking synaptotagmin. Cell 73:1291-1305.

Pierce SB, Costa M, Wisotzkey R, Devadhar S, Homburger SA, Buchman AR, Ferguson KC, Heller J, Platt DM, Pasquinelli AA, Liu LX, Doberstein SK, Ruvkun G (2001) Regulation of DAF-2 receptor signaling by human insulin and ins-1, a member of the unusually large and diverse C. elegans insulin gene family. Genes Dev 15:672-686.

Rand JB, Nonet ML (1997) Neurotransmitter assignments for specific neurons. In: C. elegans II (Riddle DL, Blumenthal T, Meyer BJ, Preiss JR, eds), pp 1049-1052. Cold Spring Harbor, NY: Cold Spring Harbor Laboratory.

Renden R, Berwin B, Davis W, Ann K, Chin CT, Kreber R, Ganetzky B, Martin TF, Broadie K (2001) Drosophila CAPS is an essential gene that regulates dense-core vesicle release and synaptic vesicle fusion. Neuron 31:421-437.

Rettig J, Neher E (2002) Emerging roles of presynaptic proteins in $\mathrm{Ca}^{2+}$ triggered exocytosis. Science 298:781-785.

Richmond JE, Davis WS, Jorgensen EM (1999) UNC-13 is required for synaptic vesicle fusion in C. elegans. Nat Neurosci 2:959-964.

Richmond JE, Weimer RM, Jorgensen EM (2001) An open form of syntaxin bypasses the requirement for UNC-13 in vesicle priming. Nature 412:338-341.

Rogers C, Reale V, Kim K, Chatwin H, Li C, Evans P, de Bono M (2003) Inhibition of Caenorhabditis elegans social feeding by FMRFamiderelated peptide activation of NPR-1. Nat Neurosci 6:1178-1185.

Rupnik M, Kreft M, Sikdar SK, Grilc S, Romih R, Zupancic G, Martin TF, Zorec R (2000) Rapid regulated dense-core vesicle exocytosis requires the CAPS protein. Proc Natl Acad Sci USA 97:5627-5632.

Sadakata T, Kakegawa W, Mizoguchi A, Washida M, Katoh-Semba R, Shutoh F, Okamoto T, Nakashima H, Kimura K, Tanaka M, Sekine Y, Itohara S, Yuzaki M, Nagao S, Furuichi T (2007a) Impaired cerebellar development and function in mice lacking CAPS2, a protein involved in neurotrophin release. J Neurosci 27:2472-2482.

Sadakata T, Washida M, Iwayama Y, Shoji S, Sato Y, Ohkura T, Katoh-Semba R, Nakajima M, Sekine Y, Tanaka M, Nakamura K, Iwata Y, Tsuchiya KJ, Mori N, Detera-Wadleigh SD, Ichikawa H, Itohara S, Yoshikawa T, Furuichi T (2007b) Autistic-like phenotypes in Cadps2-knockout mice and aberrant CADPS2 splicing in autistic patients. J Clin Invest 117:931-943.

Sawin ER (1996) Genetic and cellular analysis of modulated behaviors in Caenorhabditis elegans. PhD thesis, Massachusetts Institute of Technology.

Shakiryanova D, Tully A, Hewes RS, Deitcher DL, Levitan ES (2005) Activity-dependent liberation of synaptic neuropeptide vesicles. Nat Neurosci 8:173-178.

Shakiryanova D, Tully A, Levitan ES (2006) Activity-dependent synaptic capture of transiting peptidergic vesicles. Nat Neurosci 9:896-900.

Sieburth D, Madison JM, Kaplan JM (2007) PKC-1 regulates secretion of neuropeptides. Nat Neurosci 10:49-57.

Sorensen JB (2004) Formation, stabilisation and fusion of the readily releasable pool of secretory vesicles. Pflügers Arch 448:347-362.

Speidel D, Varoqueaux F, Enk C, Nojiri M, Grishanin RN, Martin TF, Hofmann K, Brose N, Reim K (2003) A family of $\mathrm{Ca}^{2+}$-dependent activator proteins for secretion: comparative analysis of structure, expression, localization, and function. J Biol Chem 278:52802-52809.

Speidel D, Bruederle CE, Enk C, Voets T, Varoqueaux F, Reim K, Becherer U, Fornai F, Ruggieri S, Holighaus Y, Weihe E, Bruns D, Brose N, Rettig J (2005) CAPS1 regulates catecholamine loading of large dense-core vesicles. Neuron 46:75-88.

Stevens DR, Wu ZX, Matti U, Junge HJ, Schirra C, Becherer U, Wojcik SM, Brose N, Rettig J (2005) Identification of the minimal protein domain required for priming activity of Munc13-1. Curr Biol 15:2243-2248. 
Sulston J, Dew M, Brenner S (1975) Dopaminergic neurons in the nematode Caenorhabditis elegans. J Comp Neurol 163:215-226.

Tandon A, Bannykh S, Kowalchyk JA, Banerjee A, Martin TF, Balch WE (1998) Differential regulation of exocytosis by calcium and CAPS in semi-intact synaptosomes. Neuron 21:147-154.

Thomas JH (1990) Genetic analysis of defecation in Caenorhabditis elegans. Genetics 124:855-872.

Waggoner LE, Hardaker LA, Golik S, Schafer WR (2000) Effect of a neuropeptide gene on behavioral states in Caenorhabditis elegans egg-laying. Genetics 154:1181-1192.

Walent JH, Porter BW, Martin TF (1992) A novel 145 kd brain cytosolic protein reconstitutes $\mathrm{Ca}^{2+}$-regulated secretion in permeable neuroendocrine cells. Cell 70:765-775.
Wassenberg JJ, Martin TF (2002) Role of CAPS in dense-core vesicle exocytosis. Ann NY Acad Sci 971:201-209.

White JG, Southgate E, Thomson JN, Brenner S (1986) The structure of the nervous system of the nematode Caenorhabditis elegans. Philos Trans $\mathrm{R}$ Soc Lond B Biol Sci 314:1-340.

Zahn TR, Macmorris MA, Dong W, Day R, Hutton JC (2001) IDA-1, a Caenorhabditis elegans homolog of the diabetic autoantigens IA-2 and phogrin, is expressed in peptidergic neurons in the worm. J Comp Neurol 429:127-143.

Zahn TR, Angleson JK, MacMorris MA, Domke E, Hutton JF, Schwartz C, Hutton JC (2004) Dense-core vesicle dynamics in Caenorhabditis elegans neurons and the role of kinesin UNC-104. Traffic 5:544559. 